\title{
BMX/Etk promotes cell proliferation and tumorigenicity of cervical cancer cells through PI3K/AKT/mTOR and STAT3 pathways
}

\author{
Yuanyuan $\mathrm{Li}^{1}$, Nan Cui ${ }^{1}$, Peng-Sheng Zheng ${ }^{1,2}$ and Wen-Ting Yang ${ }^{1}$ \\ ${ }^{1}$ Department of Reproductive Medicine, First Affiliated Hospital of Xi'an Jiaotong University, Xi'an, People's Republic of China \\ ${ }^{2}$ Section of Cancer Stem Cell Research, Key Laboratory of Environment and Genes Related to Diseases, Ministry of Education \\ of The People's Republic of China, Xi'an, People's Republic of China
}

Correspondence to: Wen-Ting Yang, email: 04203031@stu.xjtu.edu.cn

Peng-Sheng Zheng, email: zpsheng@mail.xjtu.edu.cn

Keywords: BMX, cell proliferation, cervical carcinoma, AKT, STAT3

Received: February 15, 2017 Accepted: April 17, $2017 \quad$ Published: April 27, 2017

Copyright: Li et al. This is an open-access article distributed under the terms of the Creative Commons Attribution License 3.0 (CC BY 3.0 ), which permits unrestricted use, distribution, and reproduction in any medium, provided the original author and source are credited.

\section{ABSTRACT}

Bone marrow X-linked kinase (BMX, also known as Etk) has been reported to be involved in cell proliferation, differentiation, apoptosis, migration and invasion in several types of tumors, but its role in cervical carcinoma remains poorly understood. In this study, we showed that BMX expression exhibits a gradually increasing trend from normal cervical tissue to cervical cancer in situ and then to invasive cervical cancer tissue. Through BMX-IN-1, a potent and irreversible BMX kinase inhibitor, inhibited the expression of BMX, the cell proliferation was significantly decreased. Knockdown of BMX in HeLa and SiHa cervical cancer cell lines using two different silencing technologies, TALEN and shRNA, inhibited cell growth in vitro and suppressed xenograft tumor formation in vivo, whereas overexpression of $B M X$ in the cell line C-33A significantly increased cell proliferation. Furthermore, a mechanism study showed that silencing BMX blocked cell cycle transit from G0/G1 to S or G2/M phase, and knockdown of BMX inhibited the expression of p-AKT and p-STAT3. These results suggested that BMX can promote cell proliferation through PI3K/AKT/mTOR and STAT3 signaling pathways in cervical cancer cells.

\section{INTRODUCTION}

Cervical carcinoma is the fourth most common cause of cancer and the fourth leading cause of cancer death among women worldwide. Based on the GLOBOCAN estimates, approximately 527,600 new cases of cervical carcinoma were diagnosed, of which more than $84 \%$ occurred in developing countries, and 265,700 deaths were reported in 2012 [1]. More than $90 \%$ of cervical cancers have been found to be associated with infection with high-risk types of human papillomavirus (HPV) [2]; however, the molecular mechanisms of initiation and cervical carcinogenesis are still unclear. It has been reported that some oncogenes and transcription factors are correlated with cervical cancer and likely involved in the development and progression of cervical cancer, such as SOX2 [3], KLF4 [4], OCT4 [5] and NANOG [6].
Bone marrow X-linked kinase (BMX, also known as Etk) is an intracellular nonreceptor tyrosine kinase member of the Tec family, which includes five mammalian members: Btk (Bruton's tyrosine kinase) [7, 8], Itk (IL2 inducible $T$-cell kinase) [9], BMX/Etk [10, 11], Tec (tyrosine kinase expressed in hepatocellular carcinoma) [12] and Txk (tyrosine-protein kinase TXK) [13]. BMX has several critical domains, including a Plekstrin homology (PH) domain, Tec homology (TH) domain, Src homology 3 (SH3) domain, Src homology 2 (SH2) domain and a kinase domain (SH1) [14]. BMX can be activated by several chemokines, vascular endothelial growth factor receptors, tumor necrosis factor receptor 2, epidermal growth factor receptor, ErbB3, and integrins $[15,16]$. Through its PH domain, BMX directly binds and activates focal adhesion kinase (FAK), RhoA, and PAK1 [17-19]. Moreover, through its $\mathrm{SH}$ domain in response to cytokine stimulation, BMX can also interact with 
p53, Pim-1 and RUFY1 [20-22]. Previous research has shown that BMX can mediate cell proliferation, survival, apoptosis, migration and invasion in various cancers, such as colorectal cancer, breast cancer, prostate cancer and bladder cancer [23-29]. For instance, BMX significantly promoted cell proliferation in human breast cancer MCF7 cells. Conversely, knockdown of BMX using short interfering RNA in prostate cancer cells inhibited cell proliferation $[16,30]$, and expression of a kinase-inactive mutant BMX in MDA-MB435 breast cancer cells also suppressed cell proliferation and tumorigenicity [19].

The phosphatidylinositol 3-kinase (PI3K)/protein kinase $\mathrm{B}(\mathrm{PKB}, \mathrm{AKT}) /$ mammalian target of rapamycin (mTOR) signaling pathway regulates various cellular functions that are also critical for tumorigenesis, cell mobility, cell cycle progression, proliferation and survival and therefore is frequently abnormal in many tumors, including colorectal, ovarian, breast and other tumors $[31,32]$. Upon stimuli, PI3K converts phosphatidylinositol 4,5-bisphosphate (PIP2) to phosphatidylinositol 3,4,5-trisphosphate (PIP3), which recruits BMX, AKT and its activating kinase PDK1 through a $\mathrm{PH}$ domain. Following AKT activation, mTOR can be activated $[33,34]$. Signal transducer and activator of transcription 3 (STAT3) is a transcriptional factor that has been demonstrated to be constitutively activated in several cancers, such as breast cancer, lung, colorectal and prostate cancers. STAT3 mediates the expression of many genes in response to cell stimuli and involved in cell proliferation, apoptosis, migration, survival and tumorigenesis $[35,36]$. BMX has previously been verified as an activator of STAT3, and can phosphorylate STAT3 in vitro [37, 38].

However, the function of BMX in cervical cancer is still poorly understood. In this study, we aimed to explore the role of BMX during the development and progression of cervical cancer, and we are the first to report that BMX can promote cell proliferation and tumor formation in cervical cancer by activating PI3K/AKT and STAT3 signaling pathways.

\section{RESULTS}

\section{The expression of BMX in the normal human cervix and cervical cancerous lesions}

Although, BMX has been reported in glioblastoma stem cells and various somatic carcinomas, such as prostate cancer, breast cancer and bladder cancer $[39,40]$, the function of BMX in cervical carcinoma is still not known. To investigate whether BMX is involved in cervical carcinogenesis, the expression of BMX was detected in normal cervix (NC), cervical carcinoma in situ (CIS) and invasive cervical carcinoma (ICC) samples using immunohistochemistry (Figure 1A). The percentage of positive BMX staining was significantly increased from $26.47 \%$ (NC samples, 9/34) to $68.00 \%$
(CIS samples, 17/25) and 88.46\% (ICC samples, 46/52, Figure 1B), and the immunoreactivity score (IRS) of BMX staining was also increased from $2.441 \pm 2.286$ (NC samples) to $5.280 \pm 4.326$ (CIS samples) and 5.981 \pm 2.920 (ICC samples) (Figure 1C), indicating that BMX may be increased during the progression of human cervical carcinoma. Furthermore, a western blot was used to analyze the expression of BMX in 6 normal cervical and 7 cervical cancer tissues, all of which were selected randomly. As shown in Figure 1D, the expression of BMX was significantly higher in cervical carcinoma tissues than in normal cervical tissues (Figure 1E, $p<0.01$ ). All of these results indicated that BMX was increased in cervical carcinoma and strongly suggested that BMX must be related to cervical carcinogenesis.

\section{BMX promoted proliferation of cervical cancer cells in vitro}

Western blotting was used to detect the expression of BMX in cervical cancer cell lines, and a high level of BMX expression was observed in HeLa, SiHa, HT-3 and CaSki cells, and a low level of BMX expression was observed in C-33A cells (Figure 2A). To explore the function of BMX in cervical cancer cells, BMX-IN-1, a potent, selective, and irreversible BMX kinase inhibitor, was used to attenuate the expression of BMX in HeLa and $\mathrm{SiHa}$ cells. Western blot results showed that the expression of BMX was decreased in both BMX-IN-1-treated HeLa and SiHa cells (Figure 2B and 2D). Flow cytometry analysis was used to detect the cell proliferation, and the results shown that the percentage of APC-Brdu-positive cells in HeLa-BMX-IN-1 (26.21\%) and SiHa-BMX-IN-1 $(20.34 \%)$ was significantly lower than that in control HeLa-DMSO (38.09\%) and SiHa-DMSO (34.80\%), respectively (Figure $2 \mathrm{C}$ and $2 \mathrm{E}, p<0.001$ ). Furthermore, cell viability, as determined by an MTT assay, was much lower in BMX-IN-1 treated HeLa and SiHa cells than the control cells (Supplementary Figure 2A and 2D, $p<0.001)$. These results suggested that attenuation of the expression of BMX by BMX-IN-1 treatment attenuated the cell proliferation in HeLa and SiHa cells.

Furthermore, a recombinant BMX-TALEN plasmid was transfected into HeLa cells to knockdown BMX expression (Figure 2F and Supplementary Figure 1). The results of the cell growth curve assay revealed that the cell growth of $\mathrm{HeLa}-\mathrm{BMX}^{+-}$cells was slower than that of the HeLa-wt cells (Figure 2G), and flow cytometry analysis showed that the percentage of APC-Brdu-positive cells in HeLa-BMX ${ }^{+-}(32.94 \%)$ was lower than that in HeLawt cells $(35.33 \%)$ (Figure $2 \mathrm{H}, p<0.05)$. Moreover, the expression of BMX was also knocked down in $\mathrm{SiHa}$ cells using an shBMX plasmid (Figure 2I). Accordingly, the cell growth of SiHa-shBMX cells was also slower than that of the SiHa-shGFP cells (Figure 2J), and flow cytometry analysis showed that the percentage of APC- 
Brdu-positive cells in SiHa-shBMX (31.03\%) was lower than that in SiHa-shGFP cells $(34.05 \%)$ (Figure $2 \mathrm{~K}$, $p<0.05)$. Furthermore, cell viability, as determined by an MTT assay, was much lower in BMX-Knockdown HeLa and $\mathrm{SiHa}$ cells than the control cells (Supplementary Figure $2 \mathrm{G}$ and $2 \mathrm{H}$ ). These suggesting that knockdown of
BMX expression in cervical cancer cells can attenuate cell proliferation and viability.

Moreover, BMX was stably overexpressed in C-33A cells using a recombinant plasmid, and a western blotting assay was used to detect the expression of BMX in C-33A-AcGFP and C-33A-BMX cells (Figure 2L). The
A
Control
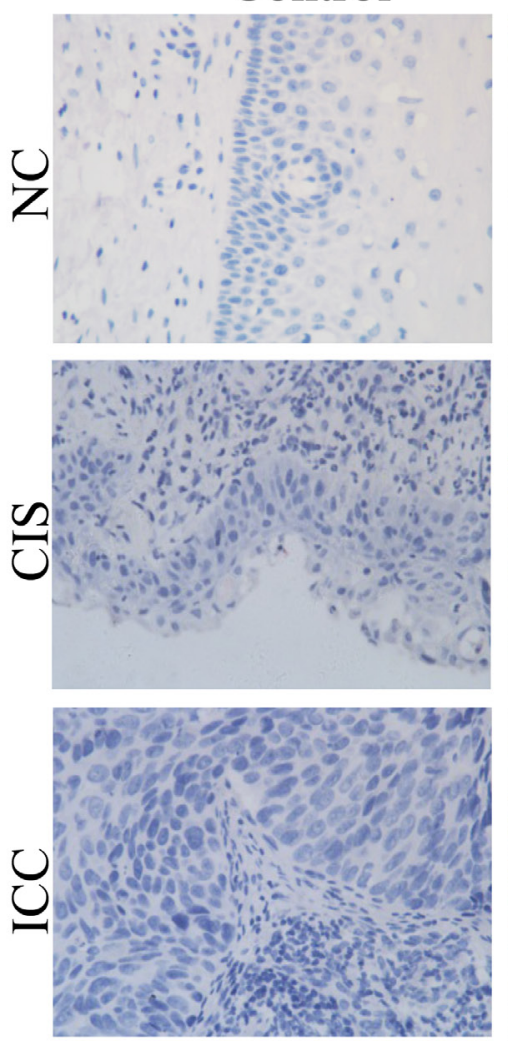

BMX
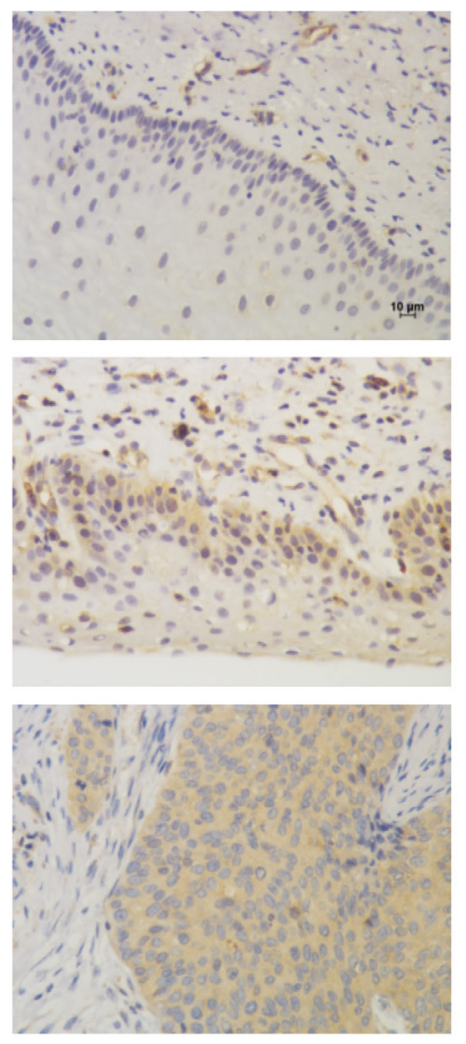

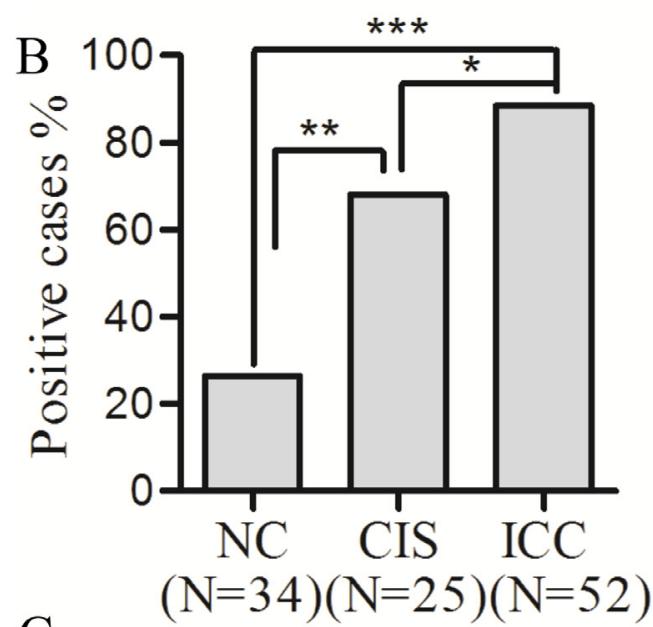

$\mathrm{C}$

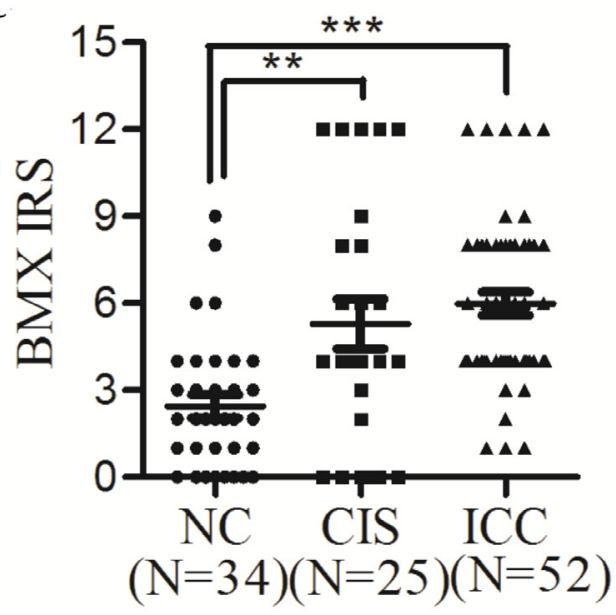

$\mathrm{D}$ $\mathrm{SCC}$

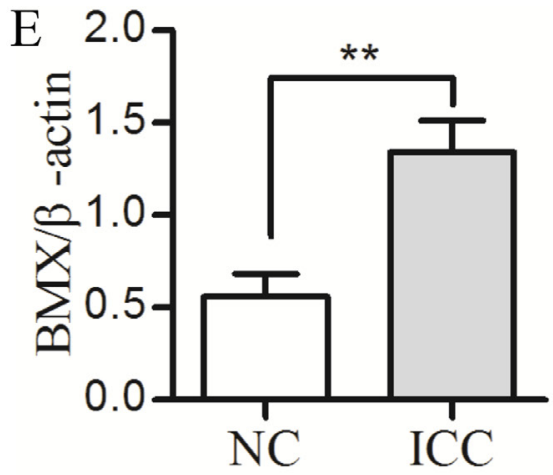

Figure 1: BMX expression is up-regulated in cervical carcinomas. (A) Immunohistochemistry (IHC) for BMX expression is shown in the normal human cervix ( $\mathrm{NC}, n=34$ ), cervical carcinoma in situ (CIS, $n=25$ ) and invasive cervical carcinoma (ICC, $n=52$ ); scale bar is $10 \mu \mathrm{m}$. (B) Analysis of the percentage of BMX-positive cells in NC, CIS and ICC using a chi-square test. (C) The average immunoreactivity score (IRS) of BMX staining in NC, CIS and ICC; one-way ANOVA was performed. (D) Western blot analysis of BMX expression in normal cervix (NC, $n=6$ ) and invasive cervical carcinoma (ICC, $n=7$ ) is shown. (E) The relative quantitative analysis of BMX expression according to western blot results using Quantity One software; a $t$-test was performed. Values are shown as the mean \pm SEM, $* p<0.05, * * p<0.01$, and $* * * p<0.001$. 
cell growth curves revealed that the cell growth of C-33ABMX cells was much faster than that of the C-33AAcGFP cells (Figure 2M), and flow cytometry analysis showed that the percentage of APC-Brdu-positive cells in C-33A-BMX (45.43\%) was higher than that in C-33AAcGFP cells $(39.95 \%)$ (Figure $2 \mathrm{~N}, p<0.05)$. Cell viability was also much higher in C-33A-BMX cells than C-33AAcGFP cells (Supplementary Figure 2I). All of these data indicated that BMX could promote the proliferation of cervical carcinoma cells.

\section{BMX promoted tumor formation of cervical cancer cells in vivo}

To investigate the effect of BMX on tumor formation in vivo, $1 \times 10^{6}$ control and $\mathrm{BMX}$-modified cells were implanted into female nude mice. As shown in Figure 3, the volume of tumors that formed from BMXknockdown cells $\left(\mathrm{HeLa}-\mathrm{BMX}^{+/-}\right.$and $\left.\mathrm{SiHa}-\mathrm{shBMX}\right)$ was much less than that of tumors formed from the control cells (HeLa-wt and SiHa-shGFP) (Figure 3A and 3C). The average weight of xenografted tumors that formed from BMX-knockdown cells (HeLa-BMX ${ }^{+/-}$and $\left.\mathrm{SiHa}-\mathrm{shBMX}\right)$ was also less than that of tumors formed from the control cells (HeLa-wt and SiHa-shGFP) (Figure 3B and 3D). However, both the C-33A-AcGFP and C-33A-BMX cells failed to form xenografted tumors in female nude mice. These results suggested that BMX can promote tumor formation of cervical cancer cells in vivo.

To determine whether BMX enhanced tumor formation by promoting cell proliferation, immunohistochemistry was used to detect the expression of BMX and Ki67 (a well-known cell proliferation maker) in the xenografted tumors. As shown in Figure 3E, the expression of BMX and $\mathrm{Ki} 67$ in the xenografted tumors that formed from HeLa-BMX ${ }^{+/}$and $\mathrm{SiHa}$-shBMX cells was much less than that observed in tumors formed from the control cells (HeLa-wt and SiHa-shGFP cells). These data suggested that BMX promoted tumor formation of cervical cancer cells in vivo, which must be dependent on the effect of BMX on cell proliferation.

\section{BMX promoted cell proliferation by accelerating cell cycle transition from $\mathrm{G} 0 / \mathrm{G} 1$ to $\mathrm{S}$ or $\mathrm{G} 2 / \mathrm{M}$ phase}

To investigate how BMX protein promoted cell proliferation, flow cytometry analysis was used to examine the changes of cell cycle in the BMX-modified cells and control cells. Treating HeLa and SiHa cells with BMXIN-1, the number of cells in G0/G1 phase was much higher in the HeLa-BMX-IN-1 (50.46\%) and SiHa-BMXIN-1 cells $(68.89 \%)$ than in the HeLa-DMSO (42.52\%) and SiHa-DMSO groups (55.89\%) (HeLa, Figure 4A and $4 \mathrm{~B}, p<0.01$, SiHa, Figure $4 \mathrm{C}$ and $4 \mathrm{D}, p<0.001$ ), while the number of cells in $\mathrm{G} 2 / \mathrm{M}$ phase was lower in the HeLa-BMX-IN-1 cells (22.91\%) and SiHa-BMX-IN-1 cells $(16.20 \%)$ than in HeLa-DMSO $(29.16 \%)$ and SiHaDMSO groups (22.12\%), respectively (HeLa, Figure 4A and 4B, $p<0.01$, SiHa, Figure 4C and 4D, $p<0.05$ ). Furthermore, the proportion of cells in $\mathrm{G} 0 / \mathrm{G} 1$ phase was also higher in the HeLa-BMX ${ }^{+-}$cells $(52.18 \%)$ than in the HeLa-wt cells $(45.91 \%, p<0.01)$, while the proportion of cells in G2/M phase was lower in the HeLa-BMX ${ }^{+/-}$ cells $(19.49 \%)$ than in the HeLa-wt cells $(25.44 \%$, Figure $4 \mathrm{E}$ and $4 \mathrm{~F}, p<0.01)$. Moreover, the proportion of cells in G0/G1 phase was much higher in the SiHashBMX cells $(59.08 \%)$ than in the SiHa-shGFP cells $(54.05 \%, p<0.05)$, while the proportion of cells in $\mathrm{G} 2 / \mathrm{M}$ phase was much lower in the SiHa-shBMX cells (18.51\%) than in the SiHa-shGFP cells $(25.63 \%$, Figure $4 \mathrm{G}$ and $4 \mathrm{H}$, $p<0.01)$. These results suggested that BMX knockdown in $\mathrm{HeLa}$ and $\mathrm{SiHa}$ cells increased the number of cells in G0/G1 phase and decreased the number of cells in $\mathrm{S}$ or $\mathrm{G} 2 / \mathrm{M}$ phase. In contrast, the proportion of cells in G0/ G1 phase was much lower in the BMX-overexpressing C-33A cells $(29.95 \%)$ than in the C-33A-AcGFP cells $(37.5 \%, p<0.01)$, while the proportion of C-33A-BMX cells in $\mathrm{G} 2 / \mathrm{M}$ phase $(27.51 \%)$ was much higher than that of the C-33A-AcGFP cells (19.92\%, Figure 4I and 4J, $p<0.01$ ), suggesting that BMX overexpression in $\mathrm{C}-33 \mathrm{~A}$ cells decreased the number of cells in $\mathrm{G} 0 / \mathrm{G} 1$ phase and increased the number of cells in $\mathrm{G} 2 / \mathrm{M}$ phase. All of these results suggested that BMX promoted the proliferation of cervical cancer cells by decreasing the number of cells in G0/G1 phase and increasing the number of cells in S or $\mathrm{G} 2 / \mathrm{M}$ phase.

\section{BMX promoted proliferation of cervical cancer cells by activating PI3K/AKT and STAT3 signaling pathways}

A previous study reported that BMX could be activated by tyrosine phosphorylation downstream of PI3K, and AKT is an essential factor in the PI3K/AKT signaling pathway, which is important for cell proliferation [41-43]. To investigate whether BMX promotes cell proliferation and tumor formation by activating AKT, a western blot was used to detect the protein level of p-AKT and AKT in HeLa-wt/HeLa-BMX ${ }^{+-}$and SiHa-shGFP/SiHa-shBMX cells. As shown in Figure $5 \mathrm{~A}$ and Supplementary Figure 5A, the expression of p-BMX and BMX was much lower in HeLa-BMX ${ }^{+-}$and SiHa-shBMX cells than in the control cells (HeLa-wt and SiHa-shGFP cells, respectively), and the expression of $\mathrm{p}$-AKT was also much lower in HeLa-BMX ${ }^{+-}$and SiHa-shBMX cells than in the control cells (HeLa-wt and SiHa-shGFP cells, respectively), while the total AKT level was not changed, suggesting that knockdown of BMX can decrease the expression of p-AKT/AKT in HeLa-BMX ${ }^{+-}$and $\mathrm{SiHa}$-shBMX cells.

Moreover, BMX has also been identified as an activator of STAT3 in glioblastoma stem cells [38]. 
A
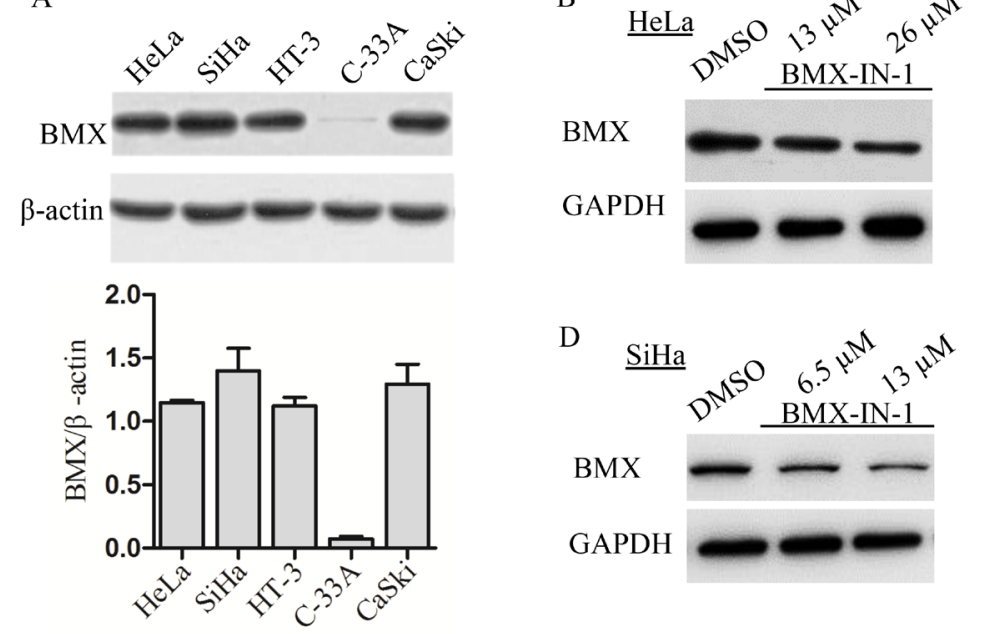

F

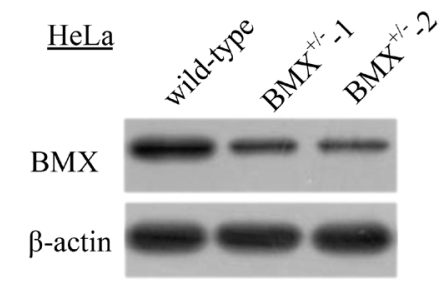

I
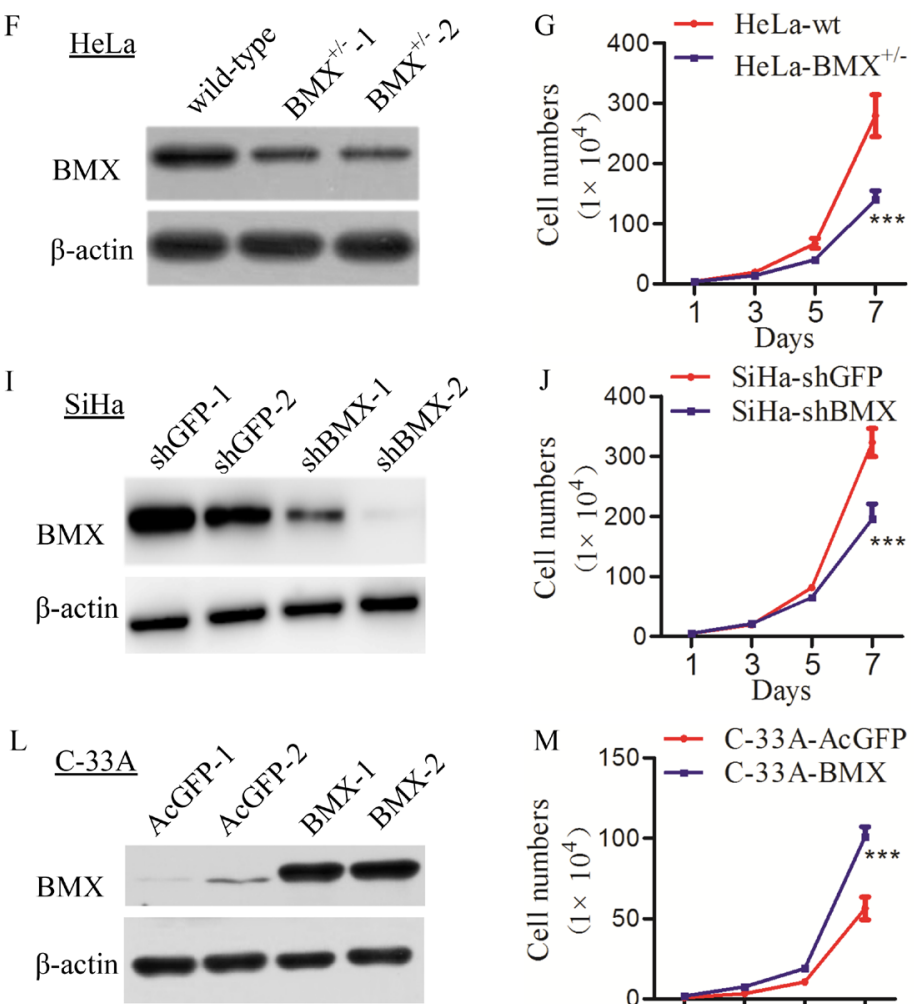

B

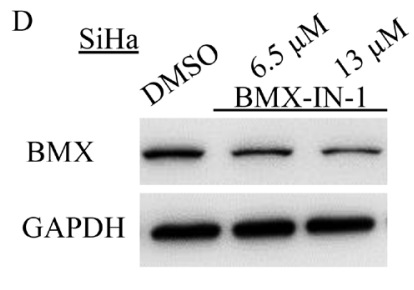

C
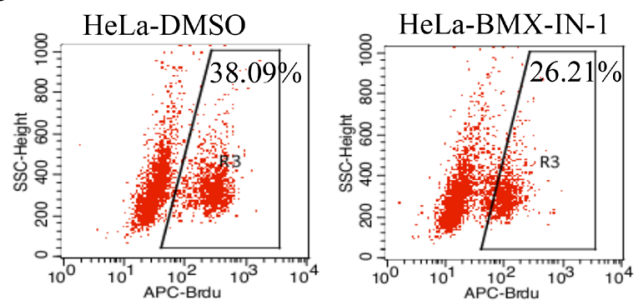

E
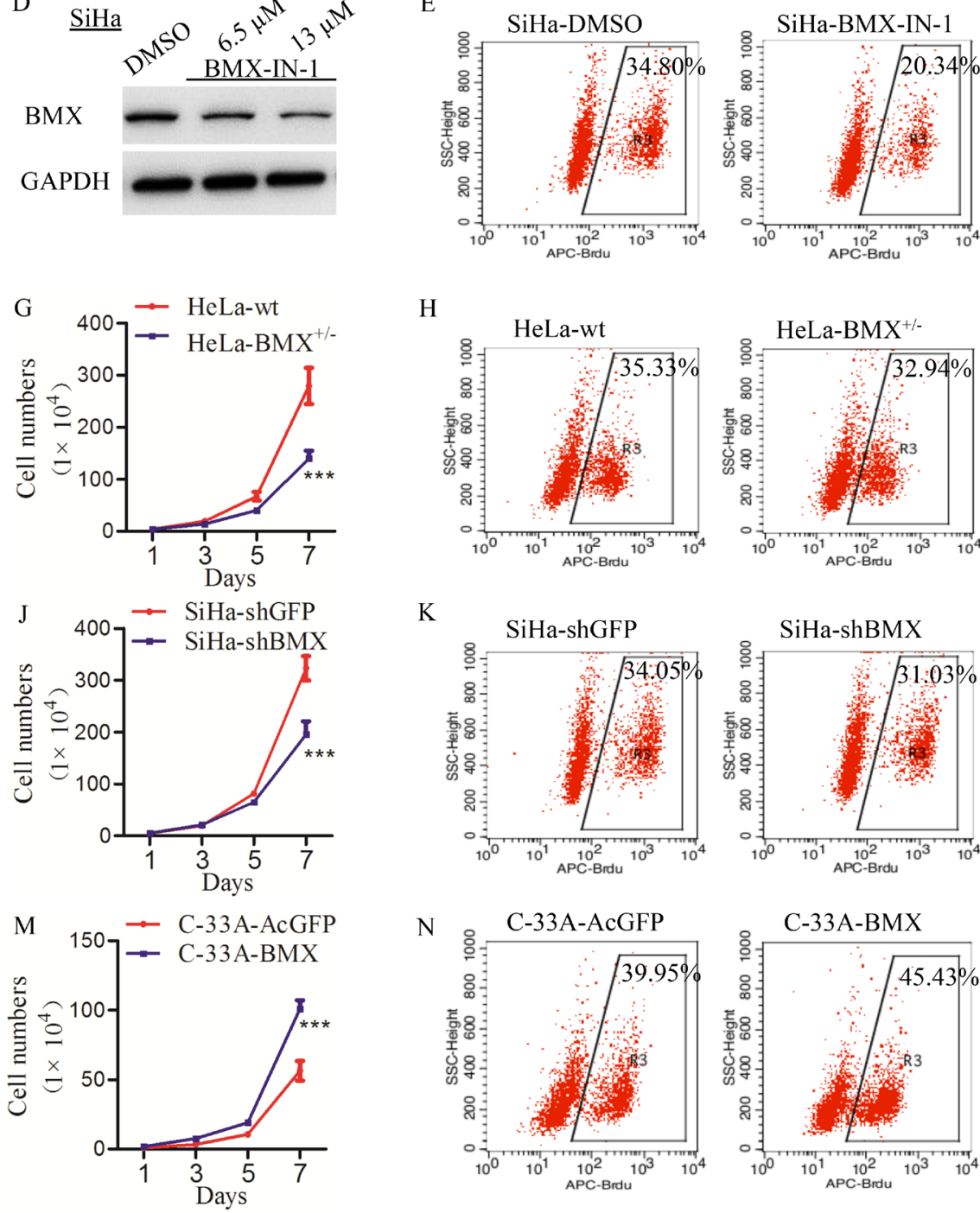

H

H
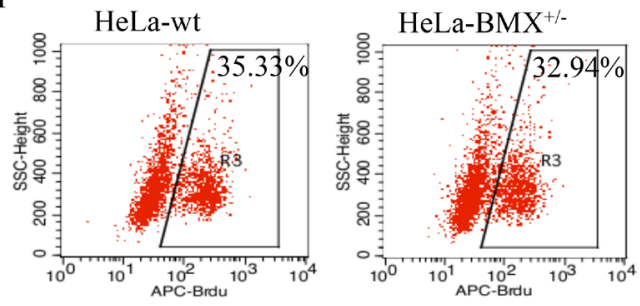

K
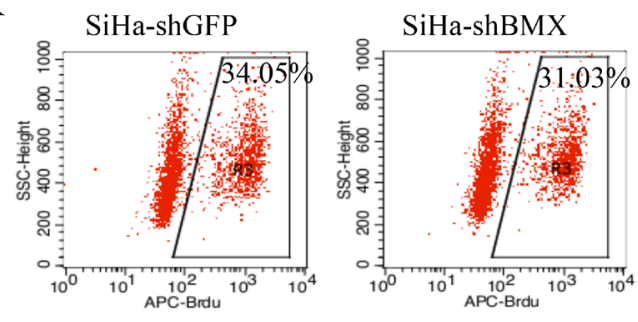

$\mathrm{N}$
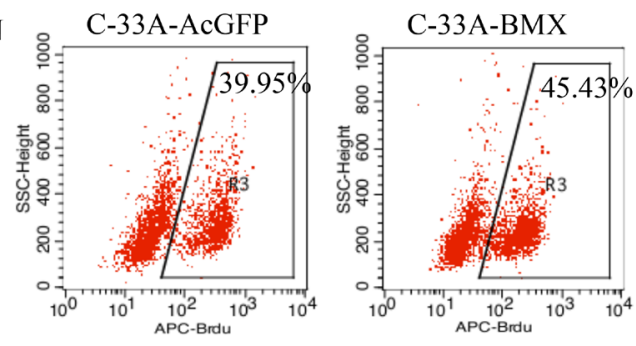

Figure 2: BMX promoted the proliferation of cervical carcinoma cells in vitro. (A) The expression of BMX in cervical cancer cell lines was detected with a western blotting assay, and the quantitative analysis using Quantity One software is shown. (B) HeLa cells were treated with DMSO as control, $13 \mu \mathrm{M}$ and $26 \mu \mathrm{M}$ BMX-IN-1, and the expression of BMX was determined using a western blotting. (C) Treated HeLa cells with DMSO, and $26 \mu \mathrm{M}$ BMX-IN-1, flow cytometry analysis was used to assess the cell proliferation with Brdu incorporation, $p<0.001$. (D) SiHa cells were treated with DMSO, $6.5 \mu \mathrm{M}$ and $13 \mu \mathrm{M}$ BMX-IN-1, and the expression of BMX was determined. (E) Treated SiHa cells with DMSO, and $13 \mu \mathrm{M}$ BMX-IN-1, the cell proliferation with Brdu incorporation was assessed, $p<0.001$. (F) A western blotting assay was used to detect the expression of BMX in TALEN-mediated HeLa BMX-knockdown clones. $(\mathbf{G})$ Growth curves and $(\mathbf{H})$ flow cytometry analysis $(p<0.05)$ were used to assess the proliferation of HeLa-wt/BMX ${ }^{+/-}$cells. $(\mathbf{I})$ A western blotting assay was used to detect the expression of BMX in shRNA-mediated SiHa BMX-knockdown clones. (J) Growth curves and (K) flow cytometry analysis $(p<0.05)$ were used to assess the proliferation of SiHa-shGFP/shBMX cells. (L) A western blotting assay was used to detect the expression of BMX in C-33A BMX-overexpressing clones (transfected with AcGFP or BMX-overexpression plasmid). (M) Growth curves and (N) flow cytometry analysis $(p<0.05)$ were used to assess the proliferation and viability of C-33A-AcGFP/BMXoverexpressing cells. Values are shown as the mean \pm SEM from three independent experiments $\left(t\right.$-test, ${ }^{*} p<0.05, * * p<0.01,{ }^{* * *} p<0.001$ vs the corresponding control). 
Constitutive activation of STAT3 accelerates cell proliferation, migration and tumor formation in several tumors, such as breast cancer and colorectal cancer. As shown in Figure 5A and Supplementary Figure 5A, the expression of p-STAT3 was much lower in both HeLa-
$\mathrm{BMX}^{+/-}$and SiHa-shBMX cells than in the control cells (HeLa-wt and SiHa-shGFP cells, respectively), while the total STAT3 level was not changed, suggesting that knockdown of BMX could also decrease the expression of p-STAT3/STAT3 in HeLa-BMX ${ }^{+-}$and SiHa-shBMX cells.

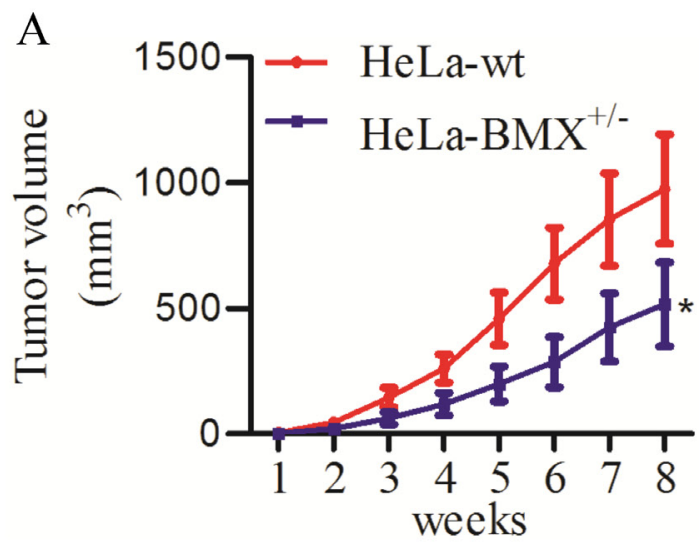

B
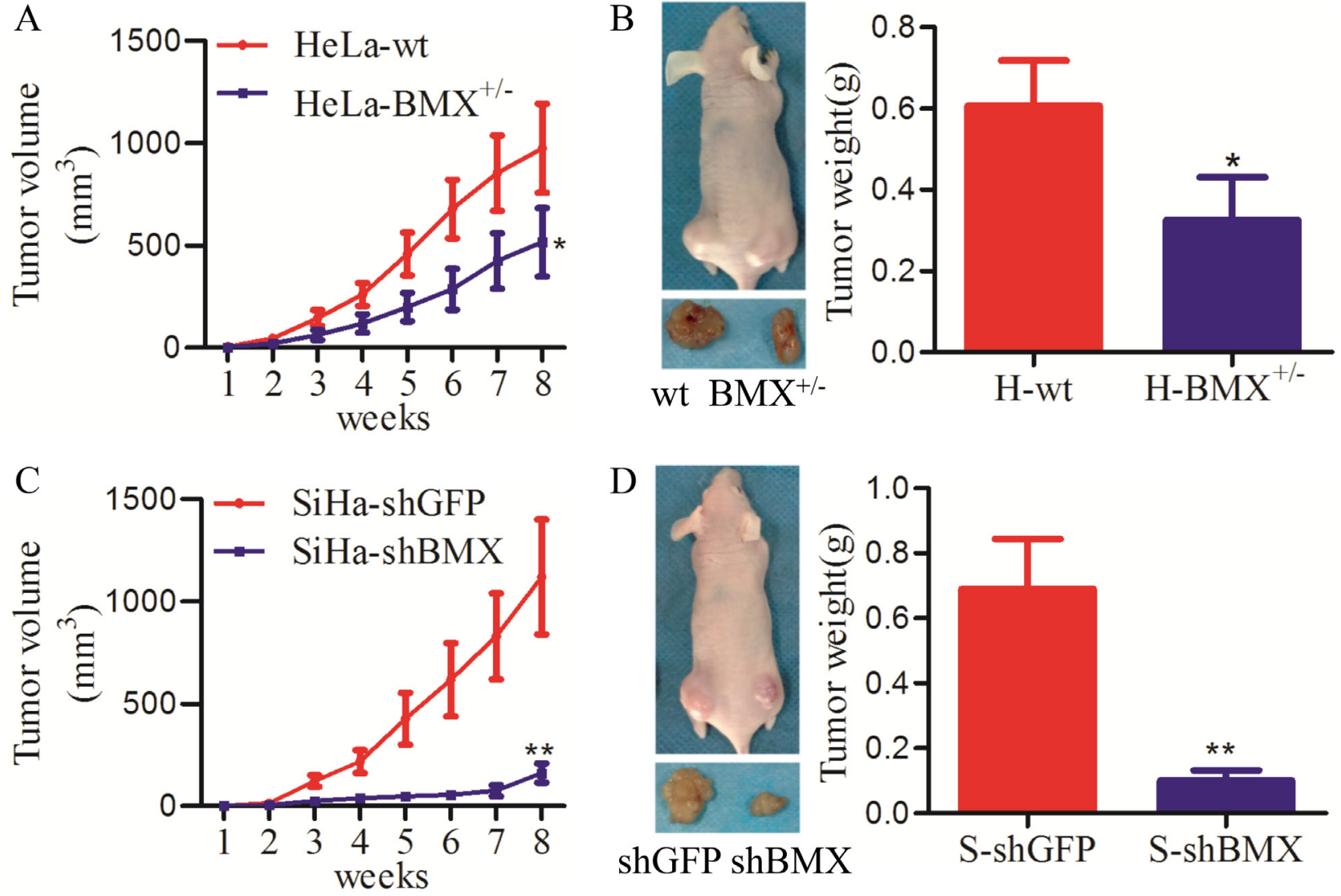

$\mathrm{D}$
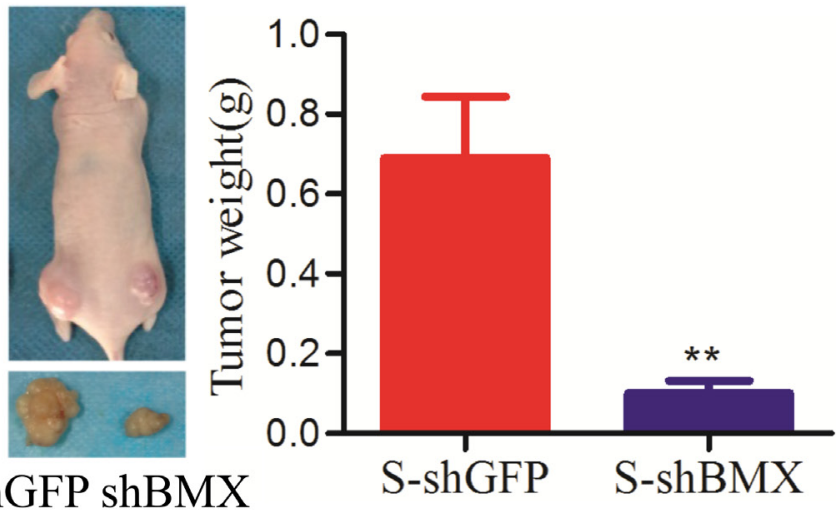

E

HeLa-wt
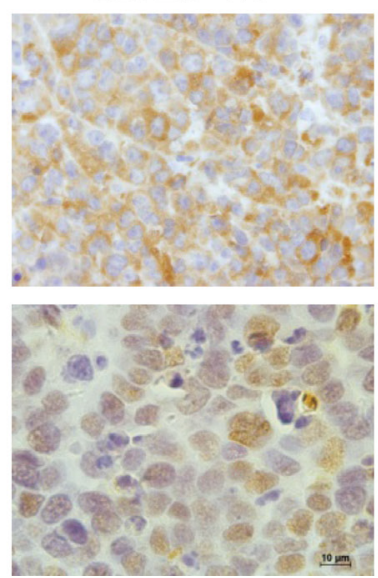

HeLa-BMX ${ }^{+/-}$
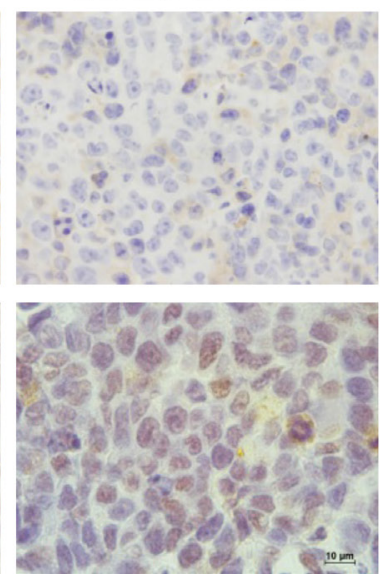

SiHa-shGFP
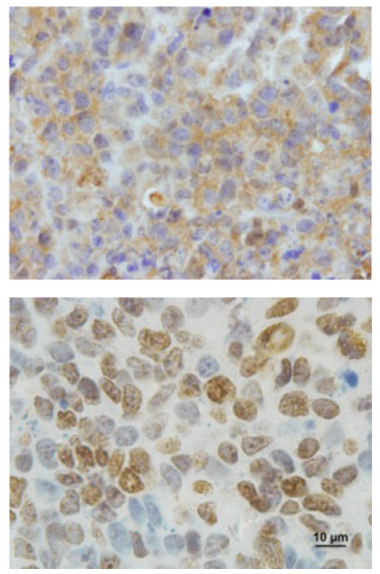

SiHa-shBMX

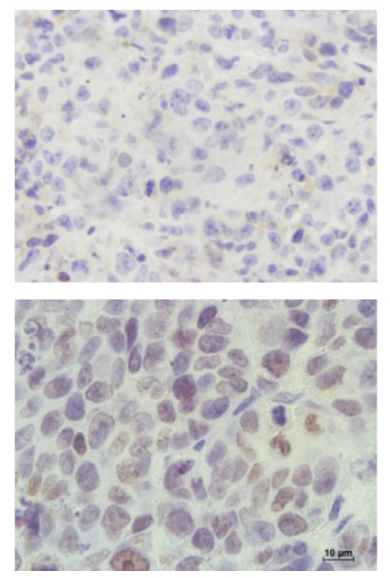

Figure 3: BMX promoted tumor growth of cervical carcinoma in vivo. (A) The tumor growth curves of BALB/c nude mice with HeLa-wt and HeLa-BMX ${ }^{+-}$cells $\left(1 \times 10^{6}\right)$ are shown. Tumors were measured weekly. $(\mathbf{B})$ Xenografts were dissociated and weighed at 8 weeks post-transplant for HeLa-wt (left) and HeLa-BMX ${ }^{+/-}$(right) cells. (C) The tumor growth curves of BALB/c nude mice with SiHa-shGFP and SiHa-shBMX cells. (D) Xenografts were dissociated and weighed at 8 weeks post-transplant for SiHa-shGFP (left) and $\mathrm{SiHa}$-shBMX (right) cells. The data were analyzed and are shown as the mean $\pm \mathrm{SEM}$. Tumor growth curves were determined using twoway ANOVA, and weights were determined using a $t$-test $(* p<0.05, * * p<0.01)$. (E) Immunochemistry of tumor xenografts stained with BMX and Ki67 is shown. Ki67 is a well-known cell proliferation marker. Scale bar $=10 \mu \mathrm{m}$. 
All of these results indicated that BMX could enhance the activity of p-AKT/AKT and p-STAT3/STAT3.

To further confirm that BMX promotes cell proliferation and tumor formation through the AKT/mTOR pathway in cervical cancer cells, the AKT inhibitor MK2206 and mTOR inhibitor rapamycin were used in HeLawt, HeLa-BMX ${ }^{+-}$, SiHa-shGFP and SiHa-shBMX cell lines. As shown in Figure 5B and Supplementary Figure 5B, in
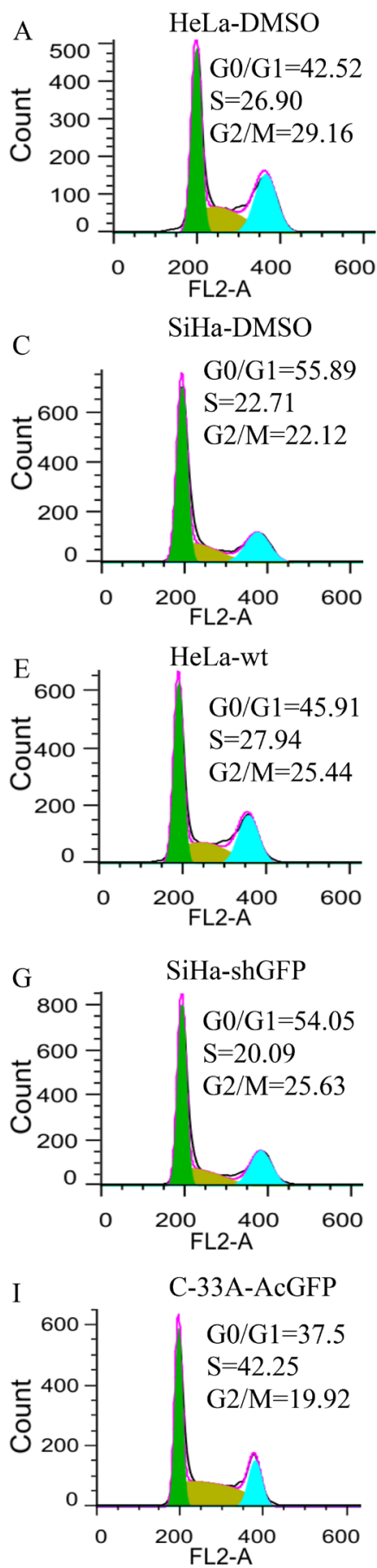

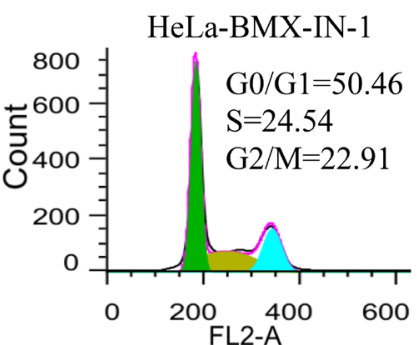

SiHa-BMX-IN-1
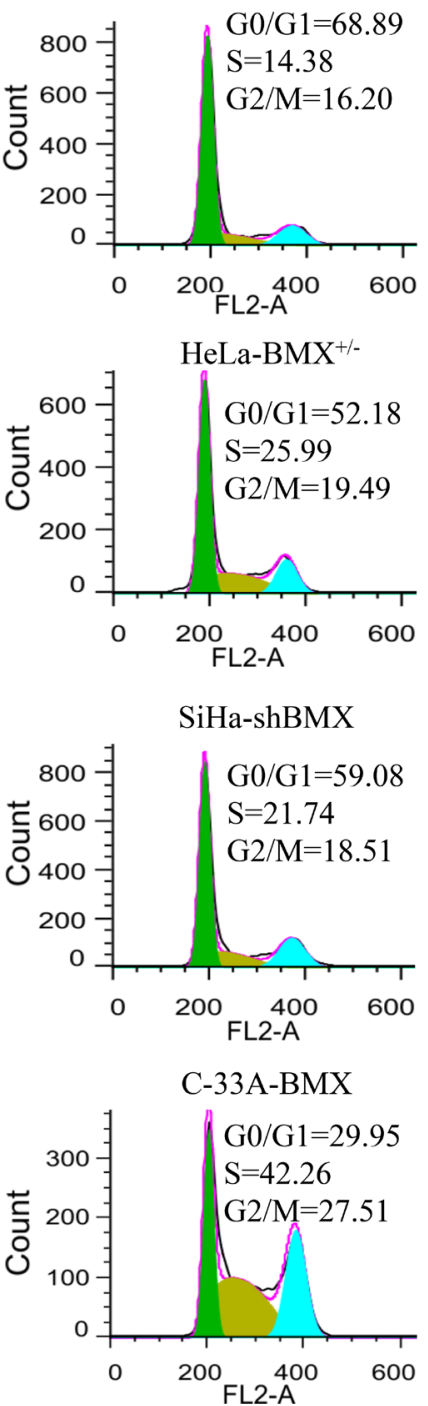

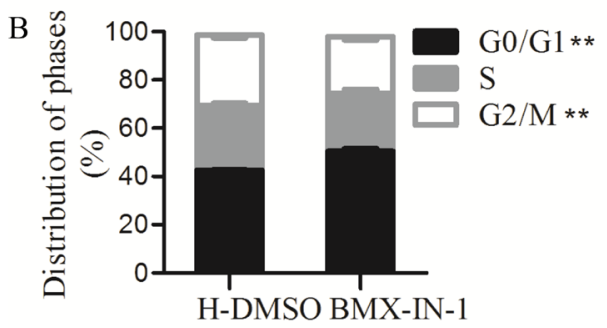

$\mathrm{D}$
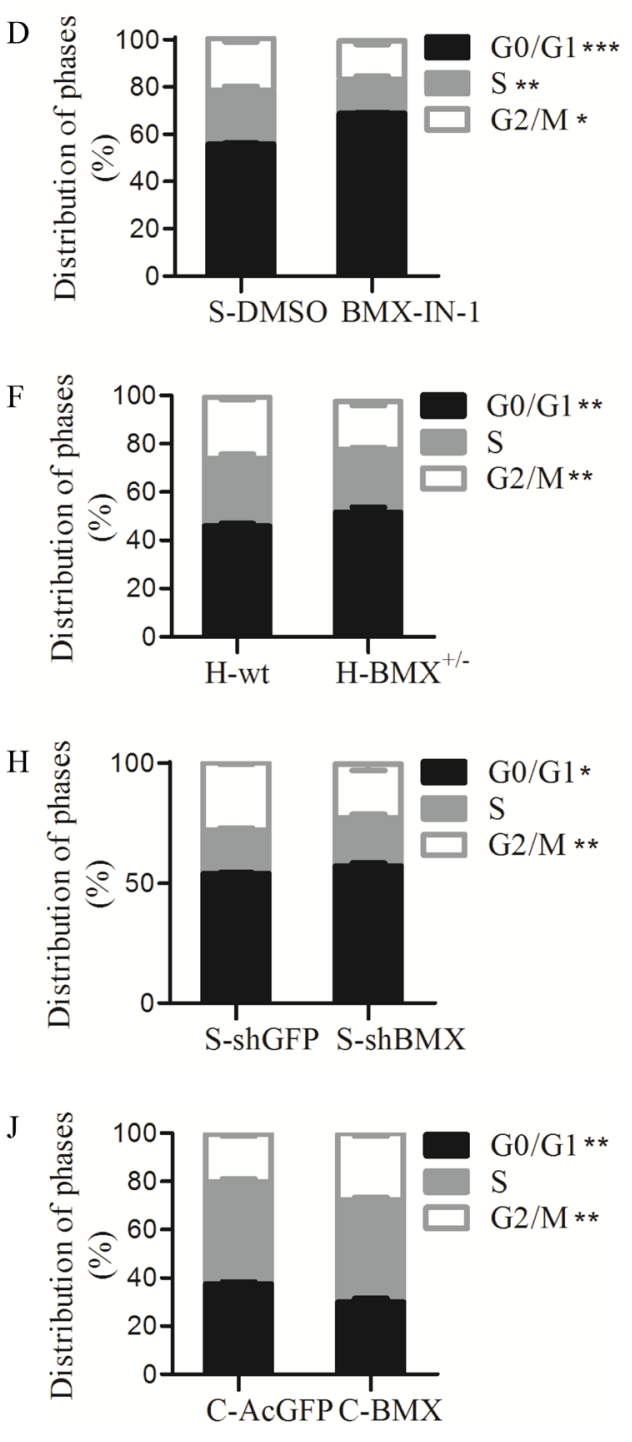

Figure 4: BMX promoted cell proliferation by accelerating cell cycle entry. (A) Treated HeLa cells with DMSO and $26 \mu \mathrm{M}$ BMX-IN-1, flow cytometry analysis was used to analyzed the cell cycle distribution. Data were processed using FlowJo 7.6 software. The statistical analysis is shown in (B). (C) Treated SiHa cells with DMSO and $13 \mu \mathrm{M}$ BMX-IN-1, and analyzed the cell cycle distribution. The statistical analysis is shown in (D). (E) Cell cycle data of HeLa-wt and HeLa-BMX ${ }^{+/}$cells was processed, and the statistical analysis is shown in (F). (G) Cell cycle data of SiHa-shGFP and SiHa-shBMX cells was processed, and the statistical analysis is shown in (H). (I) Cell cycle data of C-33A-AcGFP and C-33A-BMX cells was processed, and the statistical analysis is shown in (J). A $t$-test was used for the statistical analysis, $* p<0.05, * * p<0.01$ 
DMSO-treated HeLa-wt/BMX ${ }^{+-}$cells, the expression of both p-AKT and p-mTOR was lower in HeLa-BMX ${ }^{+-}$ cells than in HeLa-wt cells; in MK-2206-treated HeLa-wt/ $\mathrm{BMX}^{+-}$cells, the expression of both $\mathrm{p}-\mathrm{AKT}$ and $\mathrm{p}-\mathrm{mTOR}$ were inhibited compared with the DMSO-treated control group; in rapamycin-treated $\mathrm{HeLa}-\mathrm{wt} / \mathrm{BMX}^{+-}$cells, the expression of $\mathrm{p}$-mTOR but not $\mathrm{p}$-AKT was inhibited compared with the DMSO-treated control group. This result verified that $\mathrm{BMX}$ can activate the phosphorylation of AKT, and mTOR, as a canonical downstream factor, was also influenced. The results of cell proliferation assays are shown in Figure 5D and 5E, of the DMSO-treated cells, the $\mathrm{HeLa}-\mathrm{BMX}^{+/}$cells grew much more slowly than the HeLa-wt cells; of the MK-2206-treated cells, the growth of the HeLa-wt and HeLa-BMX ${ }^{+/}$clones was more inhibited than the growth of the DMSO-treated control cells, respectively. As shown in Figure 5F and 5G, the growth of rapamycin-treated HeLa-wt and HeLa-BMX ${ }^{+/-}$cells was also more inhibited than that of the DMSO-treated control cells, respectively. In SiHa-shGFP/shBMX cells, we obtained the same results with a western blotting assay (Figure 5C) and with the quantitative analysis using Quantity One software (Supplementary Figure 5C). The cell proliferation of MK-2206- (Figure 5H and 5I) and rapamycin (Figure 5J and 5K)-treated SiHa-shGFP and SiHa-shBMX cells was more inhibited than that of the DMSO-treated control cells, respectively. These results suggested that suppression of p-AKT and p-mTOR expression in HeLa and SiHa cells by MK-2206 and rapamycin inhibited proliferation of cervical cancer cells and indicated that BMX promoted the proliferation of cervical cancer cells through the PI3K/AKT/mTOR pathway.

To confirm that BMX promotes cell proliferation and tumor formation through the STAT3 pathway in cervical cancer cells, the STAT3 inhibitor cryptotanshinone was used to inhibit the expression of p-STAT3 in HeLa-wt, $\mathrm{HeLa}_{\mathrm{BMX}}^{+-}$, SiHa-shGFP and SiHa-shBMX cells. As shown in Figure 6A-6D, the expression of p-STAT3 was much lower in the cryptotanshinone-treated HeLawt $/ \mathrm{BMX}^{+/-}$and $\mathrm{SiHa}$-shGFP/shBMX cells than in the DMSO-treated cells. In addition, the cryptotanshinonetreated HeLa-wt, HeLa-BMX ${ }^{+-}$(Figure 6E and 6F), SiHashGFP and SiHa-shBMX (Figure 6G and 6H) cells grew much slower than the DMSO-treated cells, suggesting that suppression of p-STAT3 expression in HeLa and $\mathrm{SiHa}$ cells by cryptotanshinone inhibited cell proliferation. These results indicated that BMX can promote the proliferation of cervical cancer cells by enhancing the activity of STAT3. All of these results suggested that BMX promotes the proliferation of cervical cancer cells by activating the PI3K/AKT/mTOR and STAT3 pathways.

\section{DISCUSSION}

Based on the various literatures, different mechanisms underlying the development of cervical carcinoma were proposed, mainly including phosphatidylinositol-3 kinase (PI3K)/Akt/mTOR [44-46], the mitogen-activated protein kinases (MAPKs) extracellular signal-related kinase (ERK1/2) [47, 48], Janus kinase 2 (JAK2)/signal transducer and activator of transcription-3 (STAT3) [49], JAK3/STAT5 [50], NF-кB [51], Wnt/ß-catenin [52], and c-Jun N-terminal kinase $(J N K) / p-38$ [53] signaling pathways. The whole of data suggests that PI3K/AKT and STAT3 are convergence points of tumorigenic pathways in cervical carcinoma. A previous study reported that overexpression of BMX in bladder cancer cells elevated the activity of AKT and STAT3, whereas knockdown of BMX had the opposite effect [54]. Vogt PK et al. reported that an interdependence between PI3K and STAT3, and BMX may be a candidate for mediating the alliance between PI3K and STAT3 [55]. However, the connection between PI3K and BMX and STAT3 has not been explored. In this study, we first reported that BMX can promote cell proliferation and tumor progression through the $\mathrm{PI} 3 \mathrm{~K} / \mathrm{AKT} / \mathrm{mTOR}$ and STAT3 pathways. As modeled in Figure 7, these findings provide new evidence of BMX function in cervical carcinogenesis.

BMX is a member of the TEC kinase family, which is the second-largest non-receptor protein tyrosine kinase family. BMX participates in the immune response and inflammation and cytokine signaling in hematopoietic cells, endocardium, and arterial endothelium, as well as in other cells. [56-59]. Moreover, BMX is expressed in several cancers and involved in cell growth, transformation, migration, survival, apoptosis and tumorigenicity $[40,54,59,60]$.

We have verified that BMX promoted the cell proliferation in vitro and tumorigenesis in vivo. Tumorigenesis study in vivo showed that knockdown of BMX significantly diminished tumor growth in immuno-deficient mice (Figure 3). However, the in vitro study exhibited the limited effect on growth inhibition (Figure $2 \mathrm{H}$ and $2 \mathrm{~K}$ ) and cell cycle arresting (Figure $4 \mathrm{E}$ and $4 \mathrm{G}$ ) when inhibition of BMX through genetic manipulation. We think main reasons of differences between the results from in vitro and in vivo are following. On one hand, the inhibition effect of BMX is not good. Compared the proliferation (Figure 2C, Supplementary Figure 2A and Figure 2E, Supplementary Figure 2D vs. Supplementary Figure $2 \mathrm{C}$ and $2 \mathrm{~F}$ ) and cell cycle (Figure 4A, 4B and Figure 4C, 4D vs. Supplementary Figure 3A, 3B and Supplementary Figure 3C, 3D) results from two inhibitors BMX-IN-1 and LFM-A13, respectively, the differences are more significantly from the data of BMX-IN-1, a potent, selective, and irreversible BMX kinase inhibitor, than LFM-A13, a pharmacologic reversible inhibitor of BMX. Choosing the $\mathrm{BMX}^{-/ \text {c clones }}$ is vital, but it's a pity that we didn't obtain $\mathrm{BMX}^{-/}$clones in cells. On the other hand, besides cell cycle changes, apoptosis also worked between the knockdown of 
BMX and the control cells (Supplementary Figure 4). The percentage of apoptosis cells was higher in the inhibition of BMX through pharmacological and genetic manipulation cells than in the corresponding control cells.
Furthermore, an AKT inhibitor (MK-2206), mTOR inhibitor (rapamycin) and STAT3 inhibitor (cryptotanshinone) were used in HeLa-wt, HeLa-BMX ${ }^{+/}$, SiHa-shGFP and shBMX cell lines. In BMX-knocked

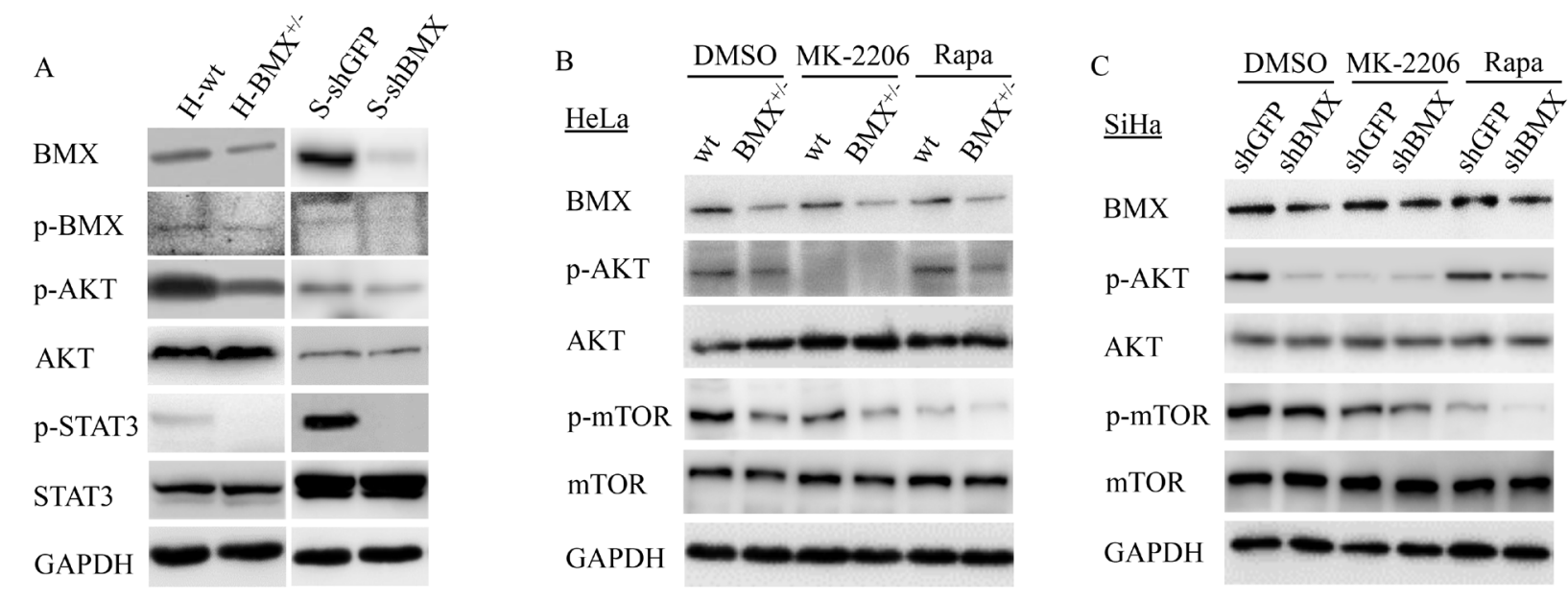

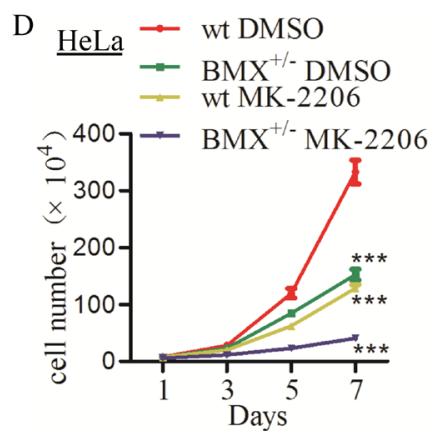

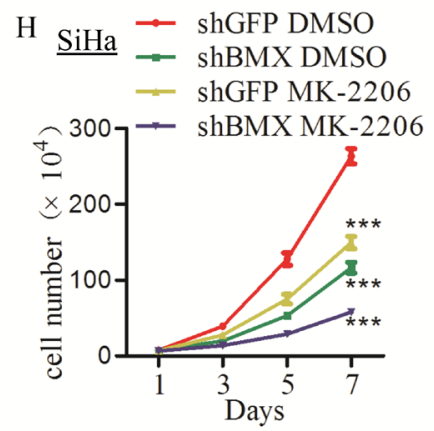

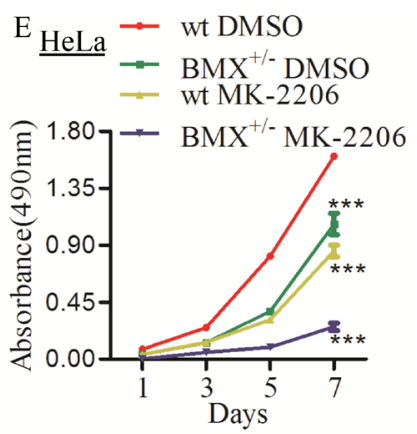
$\mathrm{F}_{\underline{\mathrm{HeLa}}} \rightarrow$ wt DMSO
$\underline{\mathrm{HeLa}}=\mathrm{BMX}^{+/-}$DMSO
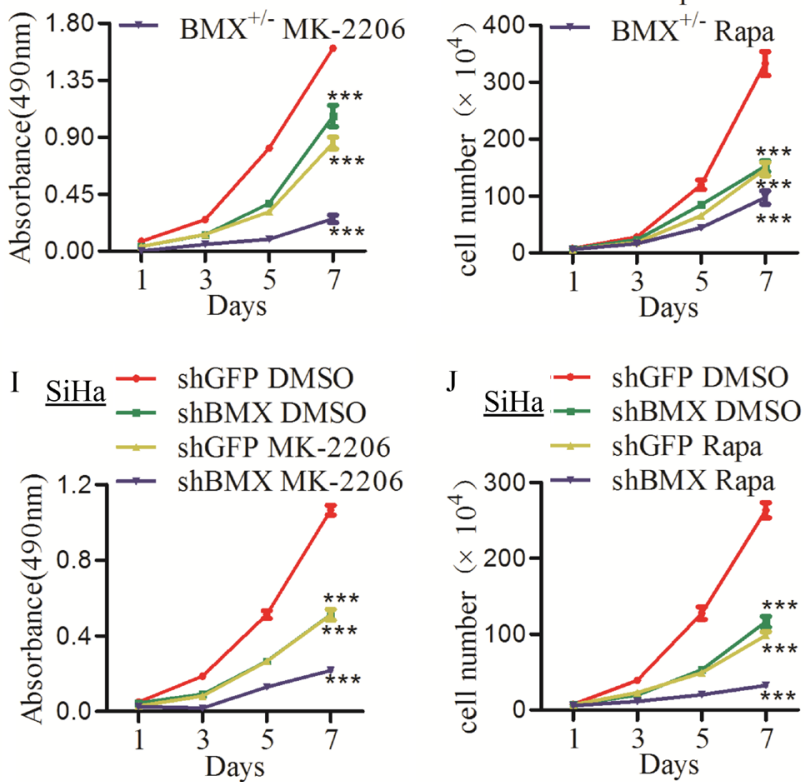

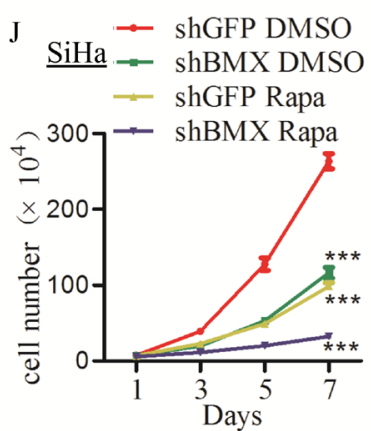

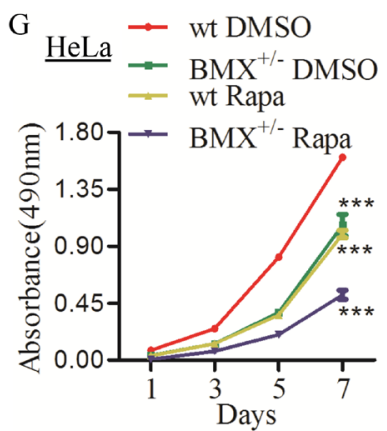

K

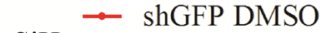

$\underline{\mathrm{SiHa}}=$ shBMX DMSO $\because$ shGFP Rapa

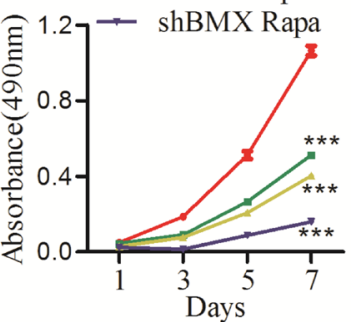

Figure 5: BMX promoted the proliferation of cervical cancer cells through the AKT/mTOR pathway. (A) Western blot detection of BMX, p-BMX, p-AKT, AKT, p-STAT3 and STAT3 in HeLa-wt/BMX ${ }^{+-}$and SiHa-shGFP/shBMX cell lines. (B) HeLa-wt/ $\mathrm{BMX}^{+-}$clones were treated with DMSO control, the selective AKT inhibitor MK-2206 $(2 \mu \mathrm{M})$ and mTOR inhibitor rapamycin $(2 \mu \mathrm{M})$, and BMX, p-AKT, AKT, p-mTOR and mTOR expression was detected using a western blotting assay. (C) SiHa-shGFP/shBMX cells were treated with DMSO control, the selective AKT inhibitor MK-2206 $(3 \mu \mathrm{M})$ and mTOR inhibitor rapamycin $(3 \mu \mathrm{M})$, and BMX, p-AKT, AKT, p-mTOR and mTOR expression was detected using a western blotting assay. (D) Growth curves and (E) MTT assays were performed to assess the proliferation and viability of HeLa-wt DMSO, BMX ${ }^{+/}$DMSO, wt MK-2206 and $\mathrm{BMX}^{+/-} \mathrm{MK}-2206$ cells. (F) The growth curves and $(\mathbf{G})$ MTT assay results of HeLa-wt DMSO, BMX ${ }^{+/-}$DMSO, wt rapamycin and BMX $^{+/-}$rapamycin cells are shown. (H) The growth curves and (I) MTT assay results of SiHa-shGFP DMSO, shBMX DMSO, shGFP MK-2206 and shBMX MK2206 cells are shown. (J) The growth curves and (K) MTT assay results of SiHa-shGFP DMSO, shBMX DMSO, shGFP rapamycin and shBMX rapamycin cells are shown. The data were compared as follows: BMX-knockdown (HeLa-BMX ${ }^{+/}$and SiHa-shBMX) groups vs. control (HeLa-wt and SiHa-shGFP) groups and all groups treated with MK-2206/rapamycin vs. DMSO-treated groups. Growth curve and MTT assay data were analyzed using two-way ANOVA, and results are shown as the mean \pm SEM. ${ }^{*} p<0.05$, $* * p<0.01, * * * p<0.001,{ }^{*} p>0.05$. 


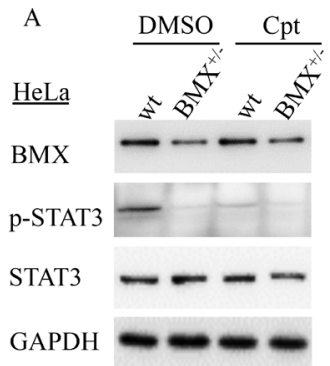

E

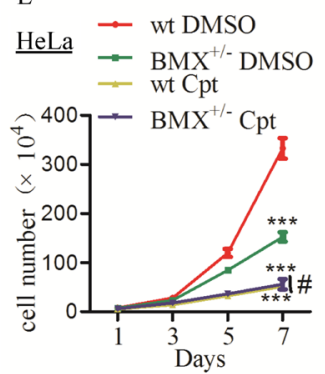

B

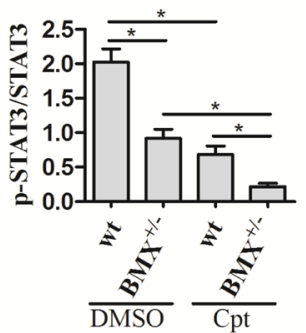

$\mathrm{F}$

$\underline{\text { HeLa }}$

$\rightarrow$ wt DMSO

$=\mathrm{BMX}^{+/-} \mathrm{DMSO}$

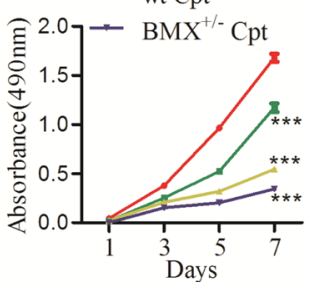

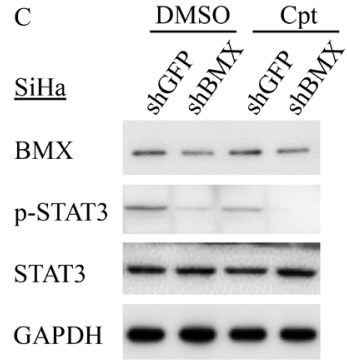

G

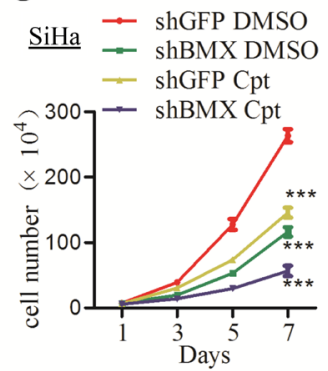

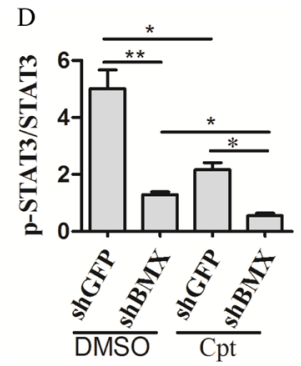

$\mathrm{H}$

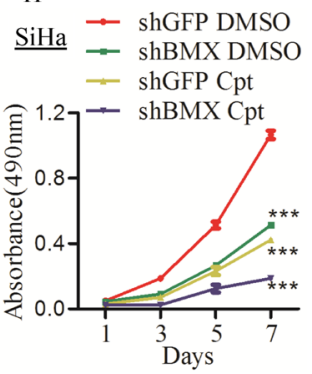

Figure 6: BMX promoted the proliferation of cervical cancer cells through the STAT3 pathway. (A) HeLa-wt/BMX ${ }^{+/}$ clones were treated with DMSO control or the STAT3 inhibitor cryptotanshinone $(2 \mu \mathrm{M})$, and BMX, p-STAT3, and STAT3 expression was detected using western blotting. The quantitative analysis of is shown in (B). (C) SiHa-shGFP/shBMX clones were treated with DMSO control or the STAT3 inhibitor cryptotanshinone $(3 \mu \mathrm{M})$, and BMX, p-STAT3, and STAT3 expression was detected using western blotting. The quantitative analysis of is shown in (D). (E) Growth curves and (F) MTT assay results for HeLa-wt DMSO, BMX ${ }^{+-}$DMSO, wt cryptotanshinone and $\mathrm{BMX}^{+/}$cryptotanshinone cells are shown. (G) Growth curves and (H) MTT assay results for SiHa-shGFP DMSO, shBMX DMSO, shGFP cryptotanshinone and shBMX cryptotanshinone cells are shown. The data were compared as follows: BMX-knockdown (HeLa-BMX ${ }^{+/}$and $\left.\mathrm{SiHa}-\mathrm{shBMX}\right)$ groups vs. control (HeLa-wt and SiHa-shGFP) groups and all groups treated with cryptotanshinone vs. the corresponding groups treated with DMSO. Western blotting data were determined using a $t$-test. Growth curve and MTT assay data were analyzed using two-way ANOVA, and are shown as the mean \pm SEM. ${ }^{*} p<0.05,{ }^{* *} p<0.01,{ }^{* * *} p<0.001,{ }^{*} p>0.05$.

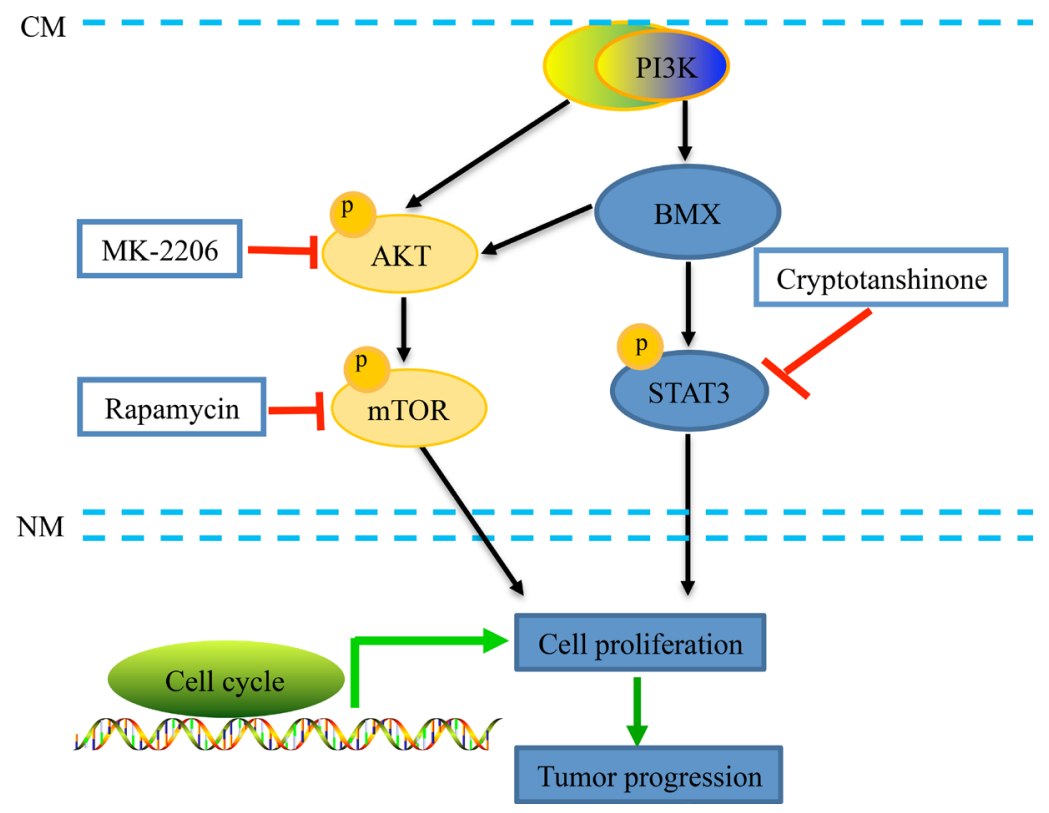

Figure 7: A schematic diagram of the BMX-mediated AKT and STAT3 pathway activation in human cervical cancer cells. Upon stimuli, BMX, as well as AKT, was activated by tyrosine phosphorylation of downstream PI3K via binding to PIP3 through the PH domain $[16,33,34]$. BMX could activate the phosphorylation of AKT and STAT3. Using three specific inhibitors of the AKT/ mTOR and STAT3 pathways, the allosteric AKT inhibitor MK-2206, mTOR inhibitor rapamycin, and STAT3 inhibitor cryptotanshinone, cell proliferation was further inhibited in BMX-knockdown groups. These findings provide new evidence of the BMX function in cell proliferation and carcinogenesis of cervical cells through the PI3K/AKT/mTOR and STAT3 pathways. 
down or silenced Hela or SiHa cells, the residual p-AKT, p-mTOR and p-STAT3 expression might be abolished by the AKT, mTOR and STAT3 specific inhibitors, respectively, thus affected further growth and survival. It could be possible that BMX cooperates with AKT/ mTOR and STAT3 to support the cell proliferation and tumorigenesis. BMX alone is not sufficient to lead normal cervical C-33A cells towards a tumorigenic phenotype. This can support the idea that BMX needs to cooperate with other pathways in vivo tumorigenesis of cervical cancer cells. In conclusion, our studies have found $\mathrm{BMX}$ can promote cell proliferation and tumorigenesis in cervical cancer cells, thus the development of BMX inhibitors, like BMX-IN-1, is necessary for cervical cancer therapy.

\section{MATERIALS AND METHODS}

\section{Cell lines and clinical samples}

The human cervical carcinoma cell lines HeLa, $\mathrm{SiHa}, \mathrm{C}-33 \mathrm{~A}, \mathrm{HT}-3$ and CaSki were purchased from the American Type Culture Collection (ATCC, USA). $\mathrm{HeLa}$, SiHa and C-33A cells were cultured in Dulbecco's Modified Eagle's Medium-high glucose (DMEM; SigmaAldrich, St Louis, Mo) supplemented with $10 \%$ fetal bovine serum (FBS; Invitrogen, Carlsbad, Calif). HT-3 and CaSki cells were cultured in McCoy's 5A and RPMI1640 (Sigma-Aldrich), respectively, supplemented with $10 \%$ FBS. All cell lines were incubated at $37^{\circ} \mathrm{C}$ in an atmosphere containing $5 \% \mathrm{CO}_{2}$.

Clinical samples including 43 normal cervix (NC), 25 cervical carcinoma in situ (CIS) and 52 invasive cervical carcinoma (ICC) samples were obtained from the First Affiliated Hospital of Xi'an Jiaotong University between 2005 to 2011. This study was approved by the Ethics Committee for the Medical College of Xi'an Jiaotong University. None of the patients had received chemotherapy, immunotherapy or radiotherapy, and all of them provided their informed consent before sample collection.

\section{Immunohistochemistry and immunocytochemistry}

Formalin-fixed, paraffin-embedded tissue samples were sliced into $4-\mu \mathrm{m}$ sections and placed in a $60^{\circ} \mathrm{C}$ incubator for 3-12 h. After deparaffinization and rehydration, antigen retrieval was performed with citrate buffer in a steam pressure cooker for $2 \mathrm{~min}$, and sections were cooled quickly to room temperature. Endogenous peroxidase was blocked with $3 \% \mathrm{H}_{2} \mathrm{O}_{2}$ and washed with PBS. The sections were incubated at $4{ }^{\circ} \mathrm{C}$ overnight with the following primary antibodies: anti-BMX (1:150, 610793, BD, USA) and anti-Ki67 (1:100, sc-23900, Santa Cruz Biotechnology). They were then incubated with secondary antibodies (Vector Laboratories, Burlingham, $\mathrm{CA}$ ) at room temperature for $20 \mathrm{~min}$, visualized with $0.05 \%$ DAB (3,3'-diaminobenzidine) and counterstained with hematoxylin. The primary antibody was replaced with PBS for the negative control.

Immunocytochemistry was performed as described above. Cells were cultured on coverslips, fixed in $4 \%$ paraformaldehyde for $30 \mathrm{~min}$ at room temperature, permeabilized with $0.2 \%$ Triton $\mathrm{X}-100$ for $20 \mathrm{~min}$, and then blocked and incubated as described above.

All slides were examined using an Olympus-CX31 microscope (Olympus, Tokyo, Japan) and scored by two investigators that analyzed five randomly selected fields at $\times 40$ or $\times 100$ magnification. BMX staining was represented using an IRS that was determined by multiplying the values for staining intensity (scored as 0 , no staining; 1 , weak staining; 2 , moderate staining; or 3, strong staining) and the values for the percentage of positive cells (scored as $0,<10 \% ; 1,10 \%-25 \% ; 2,25-50 \% ; 3,50-75 \%$; or $4,75-100 \%$ ) in each sample. BMX staining was also classified into two categories, in which an IRS of $>3$ was defined as positive and the others as negative.

\section{Western blot analysis}

Cells and clinical tissues were lysed for $30-45$ mins on ice in lysis buffer containing freshly added protease inhibitor cocktail (Roche Diagnostics, USA). After BCA quantification (Pierce, USA), the protein was added to $5 \times$ loading buffer and boiled at $95^{\circ} \mathrm{C}$ for $10 \mathrm{~min}$. Equal amounts of protein were separated by SDS-PAGE and blotted onto activated polyvinylidene difluoride membranes (Millipore, USA). After blocking, the membranes were incubated with primary antibodies overnight at $4^{\circ} \mathrm{C}$. The antibodies used were as follows: BMX (1:2000, 610793, BD), phospho-Etk (Tyr40) (1:500, \#3211, Cell Signaling Technology), AKT1 (1:500, sc5298, Santa Cruz Biotechnology), p-AKT (1:1000, \#4060, Cell Signaling Technology), mTOR (1:1000, A2445, ABclonal), p-mTOR (1:1000, AP0094, ABclonal), STAT3 (1:1000, \#9132, Cell Signaling Technology), phospho-STAT3 (Tyr705) (1:1000, \#4113, Cell Signaling Technology), $\beta$-actin and GAPDH (1:1000, Santa Cruz Biotechnology). Blots were incubated with secondary antibodies coupled to horseradish peroxidase (Thermo Fisher Scientific, USA) for $1 \mathrm{~h}$ and visualized using ECL detection (Millipore) on X-ray film. Relative quantitation was analyzed with Quantity One software.

\section{Vectors construction}

For BMX-TALEN construction, software (https:// tale-nt.cac.cornell.edu/) was used to design the TALEN genomic binding site left arm (5'-ggatacaaaatctattct-3') and right arm (5'-CTTTTGCTGTGATCTTTT-3'), and 
the target sequence between the two binding sites was 16 bp in length (Supplementary Figure 1A). Different TAL repeat modules were added in accordance with the order and linked into the TALEN vectors (L15 and R11) using a PCR-based protocol according to instructions provided by SiDanSai Biotechnology Co., Ltd (Shanghai, China). The DNA sequences of IRES, AcGFP and DsRed were amplified using PCR primers: IRESFXhoI (5'-ATTCTCGAGTTATCCGCCCCTCTCCCTC-3'), IRE -SRXbaI (5'-GCTCTAGATGTGGCCATATTATCA TCG-3'), AcGFPFXbaI (5'-GCTCTAGAACCATGGTGA GCAAGGGCG-3'), AcGFPRApaI (5'-GTTGGGCCCG CTCACTTGTACAGCTCAT-3'), DsRedFXbaI (5'-GCTC TAGAACCATGGCCTCCTCCGAGA-3') and DsRed RApaI (5'-GTTGGGCCCGCTACAGGAACAGGTGGT-3') and then ligated into the preliminary BMX-TALEN plasmids to generate two new plasmids (BMX-TALENL15-IREA-AcGFP and BMX-TALEN-R11-IREA-DsRED, Supplementary Figure 1B). All constructs were verified by sequencing.

The BMX-specific short hairpin RNA (shBMX) and control shRNA GFP was purchased from GenePharma Co., Ltd. (Shanghai, China).

For the BMX-overexpression plasmid, full-length BMX cDNA was amplified from the plasmid pcDNA3.1BMX wt (a kind gift from Professor Chi-Ying F. Huang). The primer sequences were designed as follows: BMXF (5'-3')- CGCGTCGACATGGATACAAAATCTATTCTA; and BMXR (5'-3')-CTAGGTACCTCAATGCTTGTCT TTTTCCCG. The product was cleaved with SalI and $K p n I$ and ligated into the vector pCAG-IRES2-AcGFP1 (Clontech, Mountain View, Calif) to generate the pCAGIRES2-AcGFP-BMX recombinant plasmid, which was then verified by sequencing.

\section{Transfection and sorting}

After transfection of HeLa cells with the BMXTALEN vectors (left:right $=1: 1,2 \mu \mathrm{g}$ of each plasmid) using Lipofectamine 2000 reagent for 24-48 h, the cells were classified using fluorescence-activated cell sorting (FACS, BD Biosciences) based on the expression of the AcGFP and DsRed fluorescent markers. The AcGFP ${ }^{+} /$ $\mathrm{DsRED}^{+}$cells were plated on $10-\mathrm{cm}$ culture plates at a low density in growth medium for approximately 10 days. Then, individual colonies were picked and cultured. When grown to $70 \%-90 \%$ confluency, the cells were collected and lysed to extract protein and then the loss of BMX expression was confirmed using a western blotting assay to discriminate clones. We then identified candidate clones by sequencing. Briefly, genotyping at the TALEN target site was amplified for each colony using PCR $\left(98^{\circ} \mathrm{C}, 1 \mathrm{~s}\right.$; $55^{\circ} \mathrm{C}, 5 \mathrm{~s} ; 72^{\circ} \mathrm{C}, 15 \mathrm{~s}$ ) using a Thermo Scientific Phusion Human Specimen Direct PCR kit (Thermo Scientific) and a primer pair (F5'-TTTGATAAGGTGGTCTGGA-3'; R5'-AGAGGATCTTCACAGTGTA-3') designed to yield a 564 bp amplicon around the target site. Amplicons were subcloned using a TA Cloning Kit for sequencing (Life Technologies). In comparison with the other wildtype sequence, two $\mathrm{BMX}^{+/-}$mutants were characterized, which contained $2 \mathrm{bp}$ and $28 \mathrm{bp}$ deletions and harbored a frameshift mutation (Supplementary Figure 1C).

The shRNA and BMX-overexpression vector were transfected into $\mathrm{SiHa}$ and $\mathrm{C}-33 \mathrm{~A}$ cells, respectively, using Lipofectamine 2000 reagent (Invitrogen, Carlsbad, CA, USA) according to the product description. The transfected cells were selected using G418 (Calbiochem, La Jolla, CA, USA, SiHa $1000 \mu \mathrm{g} / \mathrm{mL}$; C-33A $500 \mu \mathrm{g} /$ $\mathrm{mL}$ ) for two weeks, and then, single colonies were picked, cultured and identified using western blotting.

\section{Cell proliferation and viability assays}

$1-4 \times 10^{4}$ cells were plated at the appropriate density in $35-\mathrm{mm}$ culture dishes with $2 \mathrm{~mL}$ of medium. Cells were collected, and then counted on days 1, 3, 5, and 7 using a hemocytometer. Cell growth curves were generated to measure cell proliferation.

Cell viability was measured using a 3-(4,5dimethylthiazol-yl)-2,5-diphenyltetrazolium bromide (MTT, Sigma-Aldrich) assay. Cells were plated at a density of $1-2 \times 10^{3}$ cells in 96-well plates with $200 \mu \mathrm{L}$ of medium. Following the standard protocol, the plates were assessed on days $1,3,5$, and 7 by measuring the absorbance at 490 $\mathrm{nm}$ (Bio-Rad).

Cells were seeded in complete medium containing DMSO or inhibitors (BMX-IN-1, MCE, USA; LFM-A13, Millipore and MCE, USA; MK-2206, rapamycin, cryptotanshinone, Selleck, USA) at an appropriate dose, and cell viability was measured using an MTT assay. In addition, the medium containing LFM-A13 was changed every $24 \mathrm{~h}$.

\section{Flow cytometry analysis}

Cells $\left(1 \times 10^{6}\right)$ were seeded in 60 -mm culture dishes for $24-48 \mathrm{~h}$ and labeled with $30 \mu \mathrm{M}$ Brdu for 30-60 mins. The cells were harvested, fixed, penetrated, denatured and stained with anti-APC-Brdu using APC-Brdu cell proliferation Detection Kit (KeyGEN BioTECH), then analyzed using a FACS Calibur flow cytometer (BD Biosciences, USA).

Cells were harvested when grown to $50 \%-70 \%$ confluency and fixed in $70 \%$ cold ethanol overnight at $4^{\circ} \mathrm{C}$. After being washed twice with PBS, all samples were incubated in RNase A and propidium iodide (SigmaAldrich) for $30 \mathrm{~min}$ in the dark and then analyzed using a FACS Calibur flow cytometer (BD Biosciences, USA). Cell cycle distribution was analyzed using FlowJo 7.6 software.

Apoptosis was analyzed using Annexin V-PE/7AAD Apoptosis Detection Kit (BD Biosciences, USA) according to the manufacturer's instructions. 


\section{Tumor xenograft experiment}

The animal experiments were approved by the Animal Care and Use Committee of the Medical School of Xi' an Jiaotong University. Female BALB/c nude mice (4 to 6 weeks old) were purchased from Slac Laboratory Animal Co., Ltd. (Shanghai, China) and fed in the Medical College Experimental Animal Center of Xi' an Jiaotong University. Cells $\left(1 \times 10^{6}\right)$ mixed with Matrigel (BD, USA) in $200 \mu \mathrm{L}$ total volume were injected into subcutaneous tissue (6 mice per group). The tumor size was measured weekly, and the volume was calculated using the following formula: Volume $=\left(\right.$ length $\times$ width $\left.^{2}\right) / 2$. Last, mice were killed by cervical dislocation, and tumors were dissected, weighed, fixed with $4 \%$ paraformaldehyde solution and paraffin-embedded for immunohistochemical analysis.

\section{Statistical analysis}

Statistical analyses were performed using GraphPad Prism V5.01 software (La Jolla, USA). Sample response rate was analyzed with a chi-square test. Univariate analysis was performed using Student's $t$-test (two groups) or one-way ANOVA (three or more groups). Two-factor analysis of variance was analyzed using two-way ANOVA. In all tests, a value of $p<0.05$ was defined as statistically significant, and the data are shown as the mean \pm SEM.

\section{ACKNOWLEDGMENTS AND FUNDING}

This work was supported by a grant to Prof. Peng-Sheng Zheng from the National Natural Science Foundation of China (No.81672910 and 81472728).

The BMX-overexpression plasmid (pcDNA3.1BMX wt) was a kind gift from Professor Chi-Ying F. Huang (National Yang-Ming University, Taibei).

\section{CONFLICTS OF INTEREST}

All authors declare that they have no conflicts of interest in the article.

\section{REFERENCES}

1. Torre LA, Bray F, Siegel RL, Ferlay J, Lortet-Tieulent J, Jemal A. Global cancer statistics, 2012. CA Cancer J Clin. 2015; 65:87-108.

2. Geiger T, Sabanay H, Kravchenko-Balasha N, Geiger B, Levitzki A. Anomalous features of EMT during keratinocyte transformation. PLoS ONE. 2008; 3:e1574.

3. Ji J, Zheng PS. Expression of Sox2 in human cervical carcinogenesis. Human Pathology. 2010; 41:1438-47.

4. Yang WT, Zheng PS. Krüppel-like factor 4 functions as a tumor suppressor in cervical carcinoma. Cancer. 2012; 118:3691-702.

5. Wang YD, Cai N, Wu XL, Cao HZ, Xie LL, Zheng PS. OCT4 promotes tumorigenesis and inhibits apoptosis of cervical cancer cells by miR-125b/BAK1 pathway. Cell Death and Disease. 2013; 4:e760.

6. Gu TT, Liu SY, Zheng PS. Cytoplasmic NANOG-positive stromal cells promote human cervical cancer progression. Am J Pathol. 2012; 181:652-61.

7. Tsukada S, Saffran DC, Rawlings DJ, Parolini O, Allen RC, Klisak I, Sparkes RS, Kubagawa H, Mohandas T, Quan S, Belmont JW, Cooper DW, Conley ME, Witte ON. Deficient expression of a $\mathrm{B}$ cell cytoplasmic tyrosine kinase in human X-linked agammaglobulinemia. Cell. 1993; 72:279-90.

8. Vetrie D, Vorechovsky I, Sideras P, Holland J, Davies A, Flinter F, Hammarstrom L, Kinnon C, Levinsky R, Bobrow M, Edvard Smith CI, Bentley DR. The gene involved in X-linked agammaglobulinaemia is a member of the src family of protein-tyrosine kinases. Nature. 1993; 361:226-33.

9. Yamada N, Kawakami Y, Kimura H, Fukamachi H, Baier G, Altman A, Kato T, Inagaki Y, Kawakami T. Structure and expression of novel protein-tyrosine kinases, Emb and Emt, in hematopoietic cells. Biochem Biophys Res Commun. 1993; 192:231-40.

10. Robinson D, He F, Pretlow T, Kung HJ. A tyrosine kinase profile of prostate carcinoma. Proc Natl Acad Sci U S A. 1996; 93:5958-62.

11. Tamagnone L, Lahtinen I, Mustonen T, Virtaneva K, Francis F, Muscatelli F, Alitalo R, Smith CI, Larsson C, Alitalo K. BMX, a novel nonreceptor tyrosine kinase gene of the BTK/ITK/TEC/TXK family located in chromosome Xp22.2. Oncogene. 1994; 9:3683-8.

12. Mano H, Ishikawa F, Nishida J, Hirai H, Takaku F. A novel protein-tyrosine kinase, tec, is preferentially expressed in liver. Oncogene. 1990; 5:1781-6.

13. Haire RN, Ohta Y, Lewis JE, Fu SM, Kroisel P, Litman GW. TXK, a novel human tyrosine kinase expressed in $\mathrm{T}$ cells shares sequence identity with Tec family kinases and maps to 4p12. Hum Mol Genet. 1994; 3:897-901.

14. Smith CI, Islam TC, Mattsson PT, Mohamed AJ, Nore BF, Vihinen M. The Tec family of cytoplasmic tyrosine kinases: mammalian Btk, Bmx, Itk, Tec, Txk and homologs in other species. Bioessays. 2001; 23:436-46.

15. Pan S, An P, Zhang R, He X, Yin G, Min W. Etk/Bmx as a Tumor Necrosis Factor Receptor Type 2-Specific Kinase: Role in Endothelial Cell Migration and Angiogenesis. Molecular and Cellular Biology. 2002; 22:7512-23.

16. Jiang X, Borgesi RA, McKnight NC, Kaur R, Carpenter CL, Balk SP. Activation of Nonreceptor Tyrosine Kinase Bmx/ Etk Mediated by Phosphoinositide 3-Kinase, Epidermal Growth Factor Receptor, and ErbB3 in Prostate Cancer Cells. Journal of Biological Chemistry. 2007; 282:32689-98.

17. Chen R, Kim O, Li M, Xiong X, Guan JL, Kung HJ, Chen H, Shimizu Y, Qiu Y. Regulation of the PH-domaincontaining tyrosine kinase Etk by focal adhesion kinase through the FERM domain. Nat Cell Biol. 2001; 3:439-44. 
18. Kim O, Yang J, Qiu Y. Selective Activation of Small GTPase RhoA by Tyrosine Kinase Etk through Its Pleckstrin Homology Domain. Journal of Biological Chemistry. 2002; 277:30066-71.

19. Bagheri-Yarmand R, Mandal M, Taludker AH, Wang RA, Vadlamudi RK, Kung HJ, Kumar R. Etk/Bmx Tyrosine Kinase Activates Pak1 and Regulates Tumorigenicity of Breast Cancer Cells. Journal of Biological Chemistry. 2001; 276:29403-9.

20. Jiang T, Guo Z, Dai B, Kang M, Ann DK, Kung HJ, Qiu Y. Bi-directional Regulation between Tyrosine Kinase Etk/BMX and Tumor Suppressor p53 in Response to DNA Damage. Journal of Biological Chemistry. 2004; 279:50181-9.

21. Xie Y, Xu K, Dai B, Guo Z, Jiang T, Chen H, Qiu Y. The $44 \mathrm{kDa}$ Pim-1 kinase directly interacts with tyrosine kinase Etk/BMX and protects human prostate cancer cells from apoptosis induced by chemotherapeutic drugs. Oncogene. $2005 ; 25: 70-8$.

22. Yang J, Kim O, Wu J, Qiu Y. Interaction between Tyrosine Kinase Etk and a RUN Domain- and FYVE Domaincontaining Protein RUFY1. A possible role of ETK in regulation of vesicle trafficking. Journal of Biological Chemistry. 2002; 277:30219-26.

23. Guo L, Guo Y, Xiao S. Expression of Tyrosine Kinase Etk/ Bmx and Its Relationship with AP-1- and NF-\&kappa;BAssociated Proteins in Hepatocellular Carcinoma. Oncology. 2007; 72:410-6.

24. Dai B, Chen H, Guo S, Yang X, Linn DE, Sun F, Li W, Guo Z, Xu K, Kim O, Kong X, Melamed J, Qiu S, et al. Compensatory Upregulation of Tyrosine Kinase Etk/BMX in Response to Androgen Deprivation Promotes CastrationResistant Growth of Prostate Cancer Cells. Cancer Research. 2010; 70:5587-96.

25. Creighton C, Cohen I, Maoz M, Turm H, GrisaruGranovsky S, Maly B, Uziely B, Weiss E, Abramovitch R, Gross E, Barzilay O, Qiu Y, Bar-Shavit R. Etk/Bmx Regulates Proteinase-Activated-Receptor1 (PAR1) in Breast Cancer Invasion: Signaling Partners, Hierarchy and Physiological Significance. PLoS ONE. 2010; 5: e11135.

26. Tu T, Thotala D, Geng L, Hallahan DE, Willey CD. Bone Marrow X Kinase-Mediated Signal Transduction in Irradiated Vascular Endothelium. Cancer Research. 2008; 68:2861-9.

27. Chau CH, Chen KY, Deng HT, Kim KJ, Hosoya K, Terasaki T, Shih HM, Ann DK. Coordinating Etk/Bmx activation and VEGF upregulation to promote cell survival and proliferation. Oncogene. 2002; 21:8817-29.

28. Guo L, Chen P, Zhou Y, Sun Y. Non-receptor tyrosine kinase Etk is involved in the apoptosis of small cell lung cancer cells. Experimental and Molecular Pathology. 2010; 88:401-6.

29. $\mathrm{Wu}$ YM. Proteolytic Activation of Etk/Bmx Tyrosine Kinase by Caspases. Journal of Biological Chemistry. 2001; 276:17672-8.
30. Liu F, Zhang X, Weisberg E, Chen S, Hur W, Wu H, Zhao Z, Wang W, Mao M, Cai C, Simon NI, Sanda T, Wang J, et al. Discovery of a Selective Irreversible BMX Inhibitor for Prostate Cancer. ACS Chemical Biology. 2013; 8:1423-8.

31. Morgensztern D, McLeod HL. PI3K/Akt/mTOR pathway as a target for cancer therapy. Anticancer Drugs. 2005; 16:797-803.

32. Li H, Zeng J, Shen K. PI3K/AKT/mTOR signaling pathway as a therapeutic target for ovarian cancer. Arch Gynecol Obstet. 2014; 290:1067-78.

33. Jhanwar-Uniyal M, Jeevan D, Neil J, Shannon C, Albert L, Murali R. Deconstructing mTOR complexes in regulation of Glioblastoma Multiforme and its stem cells. Adv Biol Regul. 2013; 53:202-10.

34. Leslie NR, Dixon MJ, Schenning M, Gray A, Batty IH. Distinct inactivation of PI3K signalling by PTEN and 5-phosphatases. Adv Biol Regul. 2012; 52:205-13.

35. Banerjee K, Resat H. Constitutive activation of STAT3 in breast cancer cells: A review. Int J Cancer. 2016; 138:2570-8.

36. Siveen KS, Sikka S, Surana R, Dai X, Zhang J, Kumar AP, Tan BK, Sethi G, Bishayee A. Targeting the STAT3 signaling pathway in cancer: role of synthetic and natural inhibitors. Biochim Biophys Acta. 2014; 1845:136-54.

37. Guryanova OA, Wu Q, Cheng L, Lathia JD, Huang Z, Yang J, MacSwords J, Eyler CE, McLendon RE, Heddleston JM, Shou W, Hambardzumyan D, Lee J, et al. Nonreceptor Tyrosine Kinase BMX Maintains SelfRenewal and Tumorigenic Potential of Glioblastoma Stem Cells by Activating STAT3. Cancer Cell. 2011; 19:498-511.

38. Tsai YT, Su YH, Fang SS, Huang TN, Qiu Y, Jou YS, Shih HM, Kung HJ, Chen RH. Etk, a Btk family tyrosine kinase, mediates cellular transformation by linking Src to STAT3 activation. Mol Cell Biol. 2000; 20:2043-54.

39. Dai B. Tyrosine Kinase Etk/BMX Is Up-regulated in Human Prostate Cancer and Its Overexpression Induces Prostate Intraepithelial Neoplasia in Mouse. Cancer Research. 2006; 66:8058-64.

40. Fujisawa Y, Li W, Wu D, Wong P, Vogel C, Dong B, Kung HJ, Matsumura F. Ligand-independent activation of the arylhydrocarbon receptor by ETK (Bmx) tyrosine kinase helps MCF10AT1 breast cancer cells to survive in an apoptosis-inducing environment. Biological Chemistry. $2011 ; 392$.

41. Franke TF. Intracellular signaling by Akt: bound to be specific. Sci Signal. 2008; 1: pe29.

42. Zoncu R, Efeyan A, Sabatini DM. mTOR: from growth signal integration to cancer, diabetes and ageing. Nat Rev Mol Cell Biol. 2011; 12:21-35.

43. Cantley LC. The phosphoinositide 3-kinase pathway. Science. 2002; 296:1655-7.

44. Feng T, Zheng L, Liu F, Xu X, Mao S, Wang X, Liu J, Lu Y, Zhao W, Yu X, Tang W. Growth factor progranulin 
promotes tumorigenesis of cervical cancer via PI3K/Akt/ mTOR signaling pathway. Oncotarget. 2016; 7:58381-95. doi: 10.18632/oncotarget.11126.

45. Wu J, Chen C, Zhao KN. Phosphatidylinositol 3-kinase signaling as a therapeutic target for cervical cancer. Curr Cancer Drug Targets. 2013; 13:143-56.

46. Huang L, Huang Z, Fan Y, He L, Ye M, Shi K, Ji B, Huang J, Wang Y, Li Q. FOXC1 promotes proliferation and epithelial-mesenchymal transition in cervical carcinoma through the PI3K-AKT signal pathway. Am J Transl Res. 2017; 9:1297-306.

47. Aguilar C, Aguilar C, Lopez-Marure R, Jiménez-Sánchez A, Rocha-Zavaleta L. Co-stimulation with stem cell factor and erythropoietin enhances migration of c-Kit expressing cervical cancer cells through the sustained activation of ERK1/2. Molecular Medicine Reports. 2014; 9:1895-902.

48. Engelbrecht AM, Gebhardt S, Louw L. Ex vivo study of MAPK profiles correlated with parameters of apoptosis during cervical carcinogenesis. Cancer Lett. 2006; 235:93-9.

49. Siejka A, Schally AV, Barabutis N. Activation of Janus kinase/signal transducer and activator of transcription 3 pathway by growth hormone-releasing hormone. Cellular and Molecular Life Sciences. 2009; 67:959-64.

50. Valle-Mendiola A, Weiss-Steider B, Rocha-Zavaleta L, Soto-Cruz I. IL-2 enhances cervical cancer cells proliferation and JAK3/STAT5 phosphorylation at low doses, while at high doses IL-2 has opposite effects. Cancer Invest. 2014; 32:115-25.

51. Fritz RD, Radziwill G. CNK1 promotes invasion of cancer cells through NF-kappaB-dependent signaling. Mol Cancer Res. 2010; 8:395-406.

52. Chen Q, Zheng PS, Yang WT. EZH2-mediated repression of GSK-3beta and TP53 promotes Wnt/beta-catenin signalingdependent cell expansion in cervical carcinoma. Oncotarget. 2016; 7:36115-29. doi: 10.18632/oncotarget.8741.
53. Yan L, Liu X, Yin A, Wei Y, Yang Q, Kong B. Huaier aqueous extract inhibits cervical cancer cell proliferation via JNK/p38 pathway. Int J Oncol. 2015; 47:1054-60.

54. Guo SJ, Sun F, Guo ZY, Li W, Alfano A, Chen HG, Magyar CE, Huang JT, Chai TC, Qiu SP, Qiu Y. Tyrosine Kinase ETK/BMX Is Up-Regulated in Bladder Cancer and Predicts Poor Prognosis in Patients with Cystectomy. PLoS ONE. 2011; 6.

55. Vogt PK, Hart JR. PI3K and STAT3: A New Alliance. Cancer Discovery. 2011; 1:481-6.

56. Ekman N, Lymboussaki A, Vastrik I, Sarvas K, Kaipainen A, Alitalo K. Bmx tyrosine kinase is specifically expressed in the endocardium and the endothelium of large arteries. Circulation. 1997; 96:1729-32.

57. Cenni B, Gutmann S, Gottar-Guillier M. BMX and Its Role in Inflammation, Cardiovascular Disease, and Cancer. International Reviews of Immunology. 2012; 31:166-73.

58. Gottar-Guillier M, Dodeller F, Huesken D, Iourgenko V, Mickanin C, Labow M, Gaveriaux S, Kinzel B, Mueller M, Alitalo K, Littlewood-Evans A, Cenni B. The Tyrosine Kinase BMX Is an Essential Mediator of Inflammatory Arthritis in a Kinase-Independent Manner. The Journal of Immunology. 2011; 186:6014-23.

59. Holopainen T, Lopez-Alpuche V, Zheng W, Heljasvaara R, Jones D, He Y, Tvorogov D, D'Amico G, Wiener Z, Andersson LC, Pihlajaniemi T, Min W, Alitalo K. Deletion of the endothelial Bmx tyrosine kinase decreases tumor angiogenesis and growth. Cancer Res. 2012; 72:3512-21.

60. Fox JL, Storey A. BMX Negatively Regulates BAK Function, Thereby Increasing Apoptotic Resistance to Chemotherapeutic Drugs. Cancer Research. 2015; 75:1345-55.

61. Boch J, Scholze H, Schornack S, Landgraf A, Hahn S, Kay S, Lahaye T, Nickstadt A, Bonas U. Breaking the code of DNA binding specificity of TAL-type III effectors. Science. 2009; 326:1509-12. 